\title{
Les modèles d'Allison appliqués à un Etat "à voix multiples": le Pakistan et la crise de Kargil
}

Amélie Blom

\section{(2) OpenEdition \\ 1 Journals}

Édition électronique

URL : http://journals.openedition.org/conflits/354

DOI : $10.4000 /$ conflits.354

ISSN : $1777-5345$

\section{Éditeur :}

CCLS - Centre d'études sur les conflits lilberté et sécurité, L'Harmattan

\section{Édition imprimée}

Date de publication : 1 mars 2000

ISBN : 2-7384-9385-8

ISSN : 1157-996X

\section{Référence électronique}

Amélie Blom, «Les modèles d'Allison appliqués à un Etat "à voix multiples": le Pakistan et la crise de Kargil », Cultures \& Conflits [En ligne], 37 | printemps 2000, mis en ligne le 20 mars 2006, consulté le 30 mars 2021. URL : http://journals.openedition.org/conflits/354 ; DOI : https://doi.org/10.4000/conflits. 354

Ce document a été généré automatiquement le 30 mars 2021.

Creative Commons License 


\title{
Les modèles d'Allison appliqués à un Etat "à voix multiples": le Pakistan et la crise de Kargil
}

\author{
Amélie Blom
}

Les modèles d'Allison appliqués à un Etat «à voix multiples »: le Pakistan et la crise de Kargil

Amélie BLOM ${ }^{1}$

« Minet du Cheschire » commença Alice timidement, «voudriez-vous, je vous prie, me dire quel chemin je dois prendre pour m'en aller d'ici?». "Cela dépend en grande partie du lieu où vous voulez vous rendre » répondit le Chat.

Pour le général Jehangir Karamat, ancien chef d'état-major interarmes du Pakistan ("Chief of Army Staff» ou COAS), l'armée pakistanaise se serait conduite pendant la crise de Kargil avec une incrédulité comparable à celle d'Alice au pays des merveilles² ${ }^{2}$. L'affrontement armé entre le Pakistan et l'Inde éclate le 6 mai 1999 lorsque celle-ci découvre l'infiltration de guérilleros appuyés par le Pakistan à plus de $15 \mathrm{~km}$ au-delà de la frontière disputée entre les deux pays. Ils occupent des hauteurs montagneuses entourant Kargil et Drass, deux villes situées au Ladakh, dans la partie du Cachemire que l'Inde contrôle. Deux mois auparavant pourtant, le premier ministre indien Atal Bihari Vajpayee avait été invité en grande pompe à Lahore et signait avec son homologue pakistanais une déclaration où les deux parties s'engageaient, pour la première fois depuis longtemps, à « résoudre toutes les questions, y compris celle du Jammu et Cachemire ». A cette première incohérence, s'en ajoute une autre. La crise de Kargil prendra fin le 4 juillet suivant, non par une défaite militaire mais parce que le premier ministre pakistanais Nawaz Sharif, contre l'avis de sa propre armée, accepte l'ordre de retraite unilatérale réclamé par l'Inde et imposé par les Etats-Unis. Après neuf semaines de combat, la quatrième "demi-guerre $»^{3}$ indo-pakistanaise se termine dans un concert assourdissant de critiques et de questions au Pakistan. Quelle était la rationalité de cette opération? Pourquoi l'avoir engagée? Pourquoi avoir cédé? Comment expliquer la «débâcle»? Qui, du premier ministre, de l'armée, des 
guérilleros, en est responsable ?4. Maleeha Lodhi, ambassadeur du Pakistan aux EtatsUnis de 1994 à 1997 et renommée à ce poste par les militaires après le coup d'Etat d'octobre 1999 en dépit (ou en raison ?) de son analyse particulièrement sévère de l'opération du printemps 1999, considère que «l'affaire de Kargil a exposé les vices systématiques d'un processus de prise de décision [au sommet de l'Etat pakistanais] impulsif, erratique et exagérément secret ». C'est bien en effet au niveau du processus décisionnel que se trouve la réponse aux interrogations légitimes de la presse pakistanaise. Lodhi n'hésite d'ailleurs pas à dresser le parallèle avec la crise cubaine de 1962, comparant le leadership pakistanais à celui de l'URSS d'alors. "Toute l'expérience, écrit-elle, a rappelé la critique que Mao Zedong fit alors à Kroutchev : un tel aventurisme tactique ne pouvait aboutir qu'à une capitulation stratégique $»^{5}$. On sait que dans l'analyse qu'il fit de la crise de 62, Graham Allison a salutairement déstabilisé les bases de l'analyse réaliste de la politique étrangère, opposant au mythe de l'Etat comme acteur unitaire, l'influence des contraintes structurelles et organisationnelles sur la prise de décision ${ }^{6}$. Etonnamment, ses hypothèses ont été peu appliquées à la politique étrangère des pays non occidentaux, comme si ceux-ci souffraient d'une exceptionnalité telle que le paradigme organisationnel-bureaucratique devait s'effacer devant la problématique géo-économique du «Tiers-monde $»^{7}$. B. Korany se refuse à utiliser un modèle perçu comme suspect car inspiré du processus décisionnel américain et donc culturellement connoté8. La culture serait ainsi une variable à ce point discriminante qu'elle interdirait de faire voyager les théories et les concepts de portée générale. Le culturalisme latent et inversé qui sous-tend cette critique n'est pas recevable. La déconstruction du processus décisionnel - à laquelle invitent les hypothèses d'Allison - est au contraire, de l'avis d'Ahmed Rashid, un des rares politistes pakistanais à ne pas succomber au juridicisme et à l'historicisme qui caractérisent nombre de travaux sur les relations indo-pakistanaises «sans aucun doute le premier pas pour tenter de formuler un débat plus cohérent sur la façon dont la dispute du Cachemire devrait être résolue $»^{9}$. Ce détour par l'analyse décisionnelle en dépit de la difficulté à disposer de sources précises et fiables sur une crise aussi récente - est d'autant plus justifié dans le cas de l'intervention de Kargil que celle-ci a révélé les dysfonctionnements plus structurels dont souffrait l'appareil d'Etat pakistanais. Ce sont ces dysfonctionnements qui seront l'objet central de cet article. Patents sur d'autres dossiers de politique intérieure, ils ont alors acquis une surprenante visibilité internationale, et conduiront finalement au renversement du premier ministre trois mois plus tard (le 12 octobre 1999) ${ }^{10}$. Une telle démarche permet également de cerner les limites auxquelles se heurte l'exportation du cadre d'analyse d'Allison, sans qu'il soit pour autant nécessaire de s'en remettre à un douteux relativisme culturel. Les particularités du système politique pakistanais suggèrent en effet qu'à la complémentarité, supposée par Allison lui-même, entre ses trois variables explicatives de la décision en politique étrangère (les objectifs stratégiques, le processus organisationnel, le jeu politique), il faudrait dans certains cas opposer leur hiérarchisation ; la deuxième ayant ici considérablement altéré les effets attendus des deux autres. L'analyse de la crise de Kargil invite ensuite à étoffer sa critique du modèle rationnel à l'aide d'approches éclairant la logique décisionnelle, non plus par les seuls blocages institutionnels, mais à la lumière, d'une part, des contradictions entre les avantages nationaux et les gains internationaux attendus d'une action et, d'autre part, de ses dimensions émotionnelles. Les modèles d'Allison appliqués la crise de Kargil La rationalité stratégique de l'opération Comment expliquer l'offensive pakistanaise du 
printemps 1999 contre l'Inde ? On peut, «tel Sherlock Holmes» selon l'expression d'Allison, commencer par déduire des effets même de l'action ses intentions sousjacentes, ses rationalités les plus plausibles. Il s'agit alors de postuler que la décision en politique étrangère est le fruit d'un calcul rationnel, mené par un acteur homogène (l'Etat), confronté à un nombre limité d'options pour promouvoir et défendre l'intérêt national (modèle I). C'est d'ailleurs à peu près la seule chose que l'on puisse faire sur le vif : s'en remettre à la façon dont l'enjeu est formulé par les décideurs. Ainsi, pour la très grande majorité des analyses de la presse pakistanaise au début de la crise de Kargil, l'opération pakistanaise était une réponse calculée face à un problème stratégique : l'impossibilité à altérer le rapport de force avec l'Inde dans la dispute sur le territoire du Cachemire. Ce conflit remonte à la partition de 1947, lorsque le maharadjah hindou de cet ancien Etat princier de l'Empire britannique à $80 \%$ musulman avait opté, après moult atermoiements et sous la pression coordonnée d'une forte contestation politique de son régime autoritaire et de l'avancée de tribus du Nord sur Srinagar, pour le rattachement à l'Inde plutôt qu'au Pakistan afin de sauver son règne. Le Pakistan, contestant ce choix, occupa le nord-ouest de l'Etat tandis que l'Inde envoyait ses forces armées, à la demande du souverain et saisissait l'ONU. Ce fut la première guerre indo-pakistanaise et le Cachemire est depuis lors en quelque sorte l'Alsace-Lorraine du sous-continent. Les différentes résolutions du Conseil de sécurité prévoyant la tenue d'un référendum d'autodétermination sous contrôle international pour décider si le territoire devait revenir à l'Inde ou au Pakistan n'ont jamais été appliquées (chaque Etat refusant de démilitariser sa zone en premier). Le Cachemire, juridiquement "territoire disputé ", a été l'objet de deux autres guerres (en 1965 et plus marginalement, en 1971) et reste divisé entre les deux pays par une Ligne de Contrôle (LoC) ${ }^{11}$. Le premier dilemme stratégique du Pakistan c'est donc, au terme de plus de cinquante ans d'effort, l'échec de sa politique visant à modifier le statu quo territorial. En effet, vue son infériorité conventionnelle face à l'Inde (contre laquelle elle a perdu les trois guerres), les possibilités du Pakistan en termes strictement militaires sont limitées. Selon un lieutenant-général pakistanais, le Pakistan n'a que trois options : 1/ devenir une puissance nucléaire pour faire jouer la dissuasion, 2/ aider les mouvements armés cachemiris, $3 /$ répondre localement aux attaques indiennes le long de la $\mathrm{LoC}^{12}$. La première est effective depuis les essais nucléaires de mai 1998, sans résultat politique pour autant. La deuxième option n'a pas été plus convaincante. Elle est activement menée depuis qu'en 1989 les groupes politiques cachemiris hostiles à l'« occupation indienne » ont décidé de passer à la lutte armée et lancé le «azadi movement » («mouvement pour la liberté »). Les causes profondes de ce mouvement, soulignons-le, ne doivent rien au Pakistan qui d'ailleurs avait échoué en 1965 dans sa tentative de fomenter un soulèvement populaire. Il relève avant tout de la frustration croissante d'une jeunesse cachemirie, exceptionnellement éduquée et politisée, face à la fermeture des canaux institutionnels d'expression du mécontentement, patent au lendemain des élections truquées de $1987^{13}$. Il est également la manifestation désespérée d'une revendication d'autonomie politique fort ancienne et que New Delhi a progressivement ridiculisée ${ }^{14}$. Le Pakistan a certes cherché à tirer profit de la situation, en apportant des facilités logistiques et financières aux militants cachemiris partisans du rattachement au Pakistan (ceux favorables à l'indépendance sont juste tolérés sur son sol). Il n'a pu toutefois épuiser son rival à ce point dans la guerre civile qu'il soit forcé de lui faire des concessions. La rationalité à laquelle obéissait l'opération de Kargil était en fait dictée par la troisième option qui 
d'ailleurs est clairement formalisée dans la doctrine officielle de l'armée pakistanaise de "défense offensive $»^{15}$. Le Pakistan doit être capable de donner une réponse appropriée en cas d'intensification des violations du cessez-le-feu le long de la LoC. Or, et cet aspect est souvent occulté, depuis l'été 1998 surtout, l'Inde avait intensifié ses tirs au mortier sur les villages des vallées du Neelum et du Jhelum en Azad Cachemire, pour empêcher les infiltrations de militants entraînés dans cette zone. Ceci posait au Pakistan un triple problème stratégique : la saignée de ses troupes surveillant la LoC, également visées par les tirs indiens ; le blocage de leurs routes d'approvisionnement; et enfin, « l'insoluble » problème des personnes déplacées et des réfugiés ${ }^{16}$. L'objectif de l'intervention de mai 1999 était donc bien, comme le souligne Jehangir Karamat, de créer une "vulnérabilité mutuelle le long la $\mathrm{LoC} \aleph^{17}$. Aux escalades de l'artillerie indienne sur la ligne Kel-Kotli, les Pakistanais répondraient à des kilomètres de là, sur la portion septentrionale de la LoC, mettant également l'Inde en difficulté dans la chaîne de glaciers adjacente du Siachen. Dans cette zone inhabitée jouxtant la Chine, où la LoC n'a jamais été clairement délimitée, les deux pays se livrent en effet à une guerre de position sporadique depuis qu'en 1984 l'Inde a ravi au Pakistan les positions militaires qu'il y occupait. Cette analyse stratégique de l'intervention de Kargil, si elle explique bien l'intentionnalité des décideurs, la temporalité et la localisation de la crise, ne permet pas de comprendre son évolution. Un certain nombre de faits importants doit en effet être rappelé. Une fois le pot-aux-roses découvert, l'Inde répond à la hauteur de l'humiliation que lui inflige son voisin (car l'infiltration s'est déroulée sur plusieurs mois sans que les services de renseignement indien s'en aperçoivent) et tentent de déloger «les infiltrés soutenus par le Pakistan » à coups massifs d'artillerie. Fin mai, les Pakistanais ont encore l'avantage et font perdre à l'aviation indienne, qui entre en action pour la première fois au Cachemire, deux avions et un hélicoptère. Au même moment des conversations secrètes indo-pakistanaises prévoyant un cessez-le-feu et l'établissement de "couloirs sécurisés " pour une « retraite volontaire des mujahidin » s'engagent entre les émissaires des deux premiers ministres - un journaliste indien proche de la famille de Nawaz Sharif (R. K. Mishra, président du groupe de presse Observer et ancien député du Congrès) et l'ancien secrétaire d'Etat aux affaires étrangères pakistanais, Niaz Naiik ${ }^{18}$. Le premier ministre pakistanais se rend toutefois en compagnie du COAS, le général Musharraf, dans la zone de combat côté pakistanais pour féliciter les soldats de leur bravoure. Simultanément, la plus forte concentration de troupes indiennes jamais effectuée à l'intérieur du Cachemire s'organise et des régiments lourdement armés sont déplacés tout le long de la LoC. Au cours du mois de juin, tandis que se poursuivent les discussions secrètes, les échanges à l'arme lourde continuent entre l'armée indienne et les forces régulières pakistanaises postées de l'autre côté de la LoC ainsi que les militants infiltrés. L'Inde engage parallèlement un intense bras de fer diplomatique avec l'Etat pakistanais qui, dans la deuxième quinzaine du mois de juin, affronte la pression coordonnée de l'Union européenne, du G8 et des Etats-Unis, qui le menacent de lui retirer leur aide multilatérale et en privé, le somment de retirer "ses forces $»^{19}$. La Chine, allié traditionnel du Pakistan, appelle également au respect de la LoC. Dans les premiers jours de juillet, le Congrès américain menace le Pakistan de suspendre les prêts du FMI et de la Banque mondiale (qui devaient s'engager le mois suivant). Le débat fait alors rage au Pakistan sur la nécessité ou non de poursuivre l'opération: les voix discordantes de l'armée et du gouvernement rendent la situation extrêmement confuse. Tandis que la première continue de nier son implication et de proclamer que 
Kargil est une victoire militaire des seuls « mujahidin cachemiris », le premier ministre agite le spectre d'une guerre totale et s'envole pour Washington le 3 juillet. Le lendemain, il signe avec Bill Clinton un accord imposant un retour au statu quo ante (il est ainsi obligé de reconnaître publiquement le contrôle direct du Pakistan sur les « infiltrés »). Le même jour, l'Inde annonce qu'elle a récupéré les hauteurs stratégiques de Tiger Hills. Le 9 juillet, le gouvernement pakistanais ordonne aux « mujahidin » de se retirer de la zone mais ces derniers refusent en partie d'obtempérer. C'est alors que le Pakistan subit ses plus lourdes pertes. Le 11 juillet, la cessation des hostilités est officiellement entérinée par les directeurs généraux des opérations militaires d'Inde et du Pakistan (l'armée ayant à son tour à reconnaître son implication directe). Nawaz Sharif tente alors de rejeter l'entière responsabilité du fiasco sur l'armée et se pose en sauveur de la paix victime de l'aventurisme des militaires. Opération tactiquement réussie - ayant défié les services secrets indiens et obligé l'Inde à engager son aviation - Kargil se termine donc dans le cafouillage le plus complet. Comment expliquer les ambiguïtés et les incohérences de cette intervention? Pourquoi l'armée, qui n'a délégué qu'une partie de l'opération aux mouvements islamistes armés (représentant probablement $30 \%$ des troupes), a cherché à leur en attribuer l'entier crédit? La préparation d'une opération d'une telle envergure (engagée dès janvier 99) et l'engagement à résoudre pacifiquement la question du Cachemire avec l'Inde participaient-elles d'une seule et même stratégie ? Si tel était le cas, comment expliquer alors que le Pakistan, qui avait encore l'avantage militaire, cède brutalement aux pressions internationales et ait été incapable de gérer les modalités de sortie de crise de façon consensuelle au sommet de l'Etat? Ces questions sont, au bout du compte, plus importantes que les impératifs stratégiques ayant déterminé l'opération. La primauté $\mathrm{du}$ processus organisationnel (modèle II) sur le jeu politique (modèle III) ou le syndrome de l'Etat tricéphal Le principal apport de G. Allison, on le sait, a été de montrer que les décisions internationales devaient être également analysées comme les conséquences «d'actions plus petites, souvent innombrables et conflictuelles ", initiées par un conglomérat d'organisations ayant chacune une vie propre et agissant en fonction de routines et de procédures précédemment utilisées (paradigme du processus organisationnel). En bref, que l'« acteur national » était un mythe. Il a été reproché à Allison de ne pas voir que de telles actions conflictuelles signifiaient avant tout une "défaillance de l'autorité politique ${ }^{20}$. Peut-être mais cette "défaillance» est dans bien des cas plus la norme que l'exception. En outre, on peut aussi interpréter le modèle III comme celui qui justement, bien qu'Allison ne le pose pas aussi clairement, atténue les dysfonctionnements résultant de la logique organisationnelle. En effet, les décisions de politique étrangère sont, in fine (et que ce modèle arrive en troisième position n'est pas un hasard) pour Allison le résultat de compromis, de coalitions, entre des officiels gouvernementaux dont les priorités, la capacité, les perceptions et les positions hiérarchiques détermineront l'issue du processus de marchandage. Dans le cas de la crise de Cuba, à l'issue du « jeu » entre tous les partenaires impliqués dans la prise de décision, Kennedy sut persuader l'armée, comme le département d'Etat de retenir la solution intermédiaire du blocus. Ce modèle suppose donc des acteurs prêts à «jouer ", à dialoguer, à marchander, à tenter de se persuader. Or, lors de la crise de Kargil, personne n'y était disposé. En effet, les dysfonctionnements relevant du modèle II peuvent être parfois si forts que le "marchandage » politique entre les décideurs ne peut avoir lieu. Pendant Kargil, le jeu - ou plutôt ici le «chantage " politique - entre l'armée et le premier ministre, ainsi que les fonctionnaires des Affaires étrangères 
(comme nous le verrons), ne s'est pas fait dans un bureau soustrait aux regards extérieurs mais sur le terrain, en plein jour. Les partenaires étrangers ont eu à faire à un Etat tricéphal dont les trois principaux acteurs n'étaient engagés dans aucun travail de dialogue et de persuasion réciproque. L'Etat pakistanais s'est donc conduit pendant la crise de Kargil comme un «Etat à voix multiples» («multivocal state») ${ }^{21}$. Une fois l'opération enclenchée en effet, la logique du « chacun pour soi » l'a emporté : il y a eu une absence totale de coordination entre les différents services concernés. Il est à cet égard tout à fait révélateur que le Defense Committee of the Cabinet - l'unique instance de concertation politico-militaire censée développer une politique consensuelle et, officiellement, le principal organe décisionnel en matière stratégique ${ }^{22}-$ n'ait été réuni qu'au terme de la crise et ce pour la première fois depuis presque deux ans. Le modèle organisationnel : l'armée fait ce qu'elle sait faire En dépit d'une cohérence de façade, la politique cachemirie implique une multiplicité d'intervenants: l'armée et ses deux principaux services secrets, l'Inter-Services Intelligence (ISI) et le Military Intelligence (MI) ; le secrétariat du premier ministre ; le ministère des affaires étrangères (ses deux sous-divisions «Inde » et "Cachemire »), le secrétaire d'Etat à la défense ; le ministre des affaires cachemiries; et enfin, le président et le premier ministre de l'Etat d'Azad Cashemire $^{23}$. De toutes ces institutions, celle dont le pouvoir et l'inertie ont les conséquences les plus déterminantes est, bien entendu, l'armée. Ayant dirigé le pays pendant vingt-cinq ans (de 1958 à 1972, puis de 1977 à 1988) sur les cinquante années de son existence, elle considère la politique étrangère et tout particulièrement celle à l'égard de ses voisins (Inde et Afghanistan), comme son domaine réservé. L'intervention de Kargil doit se comprendre dans le cadre de cette spécificité du système politique pakistanais. Selon la majorité des analystes de défense pakistanais, elle a été pensée, proposée et élaborée, non par le ministre de la défense (poste détenu par le premier ministre), ni même par le secrétaire d'Etat à la défense, mais par trois généraux qui occupaient des postes clés dans la hiérarchie militaire : le COAS le général Pervez Musharraf (actuel « Chef de l'exécutif »), le chef d'état-major de l'armée de terre ("Chieff of General Staff », CGS) lieutenant-général Muhammad Mir Aziz Khan, et le Commandant du 10ème corps d'armée, le lieutenant-général Mahmood Ahmed (actuellement directeur de l'ISI). Ils sont aujourd'hui les trois hommes forts du nouveau régime militaire. Dans la décision de l'opération de Kargil, comme dans sa gestion, l'armée a fait preuve d'une incontestable continuité : l'approche strictement militaire du conflit du Cachemire. Sa conviction, forgée dans le temps, de l'impossibilité de faire des compromis avec l'Inde l'explique en grande partie. Dans les années $80,80 \%$ des officiers pakistanais considéraient que le Jammu-Cachemire faisait partie intégrante du Pakistan et qu'il n'était pas négociable ${ }^{24}$. Les jeunes officiers sont également formés dans l'idée qu'il est dangereux d'établir des relations de confiance avec l'Inde, celle-ci étant fondamentalement un pays «fourbe ». De l'avis de Stephen Cohen, l'instruction reçue au Command and Staff College par exemple, peine à se départir d'une vision particulièrement rigide de la stratégie de l'Inde. Elle serait immuable car enracinée dans son communalisme hindou et sa prétention à devenir une grande puissance ${ }^{25}$. Un général à la retraite, pourtant connu pour ses positions modérées vis-à-vis de l'Inde, ne cache en effet pas en privé sa satisfaction de voir l'Inde désormais dirigé par le gouvernement nationaliste du Bharatiya Janata Party (BJP). «C'est une bonne nouvelle pour nous, dit-il, le monde verra enfin le véritable visage de l'Inde : sa déformation chauviniste hindoue $»^{26}$. Kargil a démontré "qu'en tant qu'institution, l'armée, à travers son chef d'état-major et ses commandants de corps d'armée, reste l'ultime 
garant d'une politique inflexible, sans compromis possible, vis-à-vis de l'Inde ${ }^{27}$. On ne peut sous-estimer la fonctionnalité de cette inflexibilité. En effet, le rôle dominant de l'armée en politique étrangère, comme intérieure (qu'elle contrôle par de multiples canaux), est précisément assuré par l'exagération constante - et entretenue - de la menace indienne. Il y a, de l'avis de Saeed Shafqat, une corrélation directe entre l'intervention des militaires en politique intérieure et « la manipulation des facteurs géopolitiques /.../ [en] gonflant les menaces extérieures et en les articulant avec les menaces internes $»^{28}$. Ce couplage entre l'ennemi extérieur et intérieur est particulièrement net dans la «nouvelle pensée stratégique » de l'armée : la principale menace indienne est désormais moins celle d'une attaque conventionnelle que d'une implication dissimulée dans les conflits internes qui secouent le Pakistan (violences sectaires entre groupes radicaux sunnites et chiites au Punjab, guérilla urbaine à Karachi). L'ancien COAS Karamat ici encore se fait l'écho de cette vision. L'Inde, affirme-t-il, "veut se débarrasser des irritants [sous-entendus les rebelles cachemiris] en nous affaiblissant, en nous divisant, non par une approche directe mais par le biais des conflits de basse intensité à l'intérieur du Pakistan $»^{29}$. C'est le rôle de l'armée en matière de sécurité intérieure qui se trouve ainsi justifié et de facto, en politique tout court. C'est également, et tout aussi fondamentalement, le traumatisme de la guerre de 1971 au cours de laquelle l'Inde aida le Pakistan oriental (actuel Bangladesh) dans sa guerre de sécession du Pakistan occidental (actuel Pakistan) qui se trouve réveillé. Le "parochialisme" de l'armée, pour reprendre l'expression d'Allison, explique aussi qu'elle ait sous-estimé la nécessité d'un accompagnement diplomatique. Pour Maleeha Lodhi, ce fut la plus grande erreur qu'elle commit au cours de la crise de Kargil. Considérant que l'usage de la force militaire est traditionnellement privilégié par le Pakistan lorsqu'il est incapable d'avoir une approche politique efficace et innovante, elle explique qu'en l'absence d'accompagnement diplomatique et économique, l'opération était vouée à l'échec. Selon elle, Kargil a été « un plan de guérilla conçu sans aucune pensée stratégique ni anticipation ou appréciation de ses conséquences /.../, un objectif tactique déconnecté de son environnement plus large, diplomatique, politique et économique $»^{30}$. Le Pakistan s'est en effet trouvé totalement démuni et isolé, sans soutien économique de rechange et sans discours acceptable par la "communauté internationale ", lorsque la pression diplomatique et économique s'accroissait tout au long du mois de juin. L'armée se limitait, on l'a vu, à s'en remettre aux «courageux mujahidin » qu'il fallait aider dans leur lutte pour la liberté. Ce fut le seul discours de « relations publiques » que le Pakistan opposa aux critiques du G-8, des Etats-Unis et de la Chine. Cette faille centrale de l'opération de Kargil peut s'expliquer par ce qui constitue, selon Eqbal Ahmed, une caractéristique forte de l'institution militaire pakistanaise $^{31}:$ la faiblesse de ses capacités d'expertise, attribuable en partie aux études souvent très orientées de groupes de réflexion animés par le General Headquarter de l'armée, et en partie à la priorité accordée aux faits sur l'analyse, qui relève tout autant de la dépendance vis-à-vis de l'ISI que de l'absence d'une interaction ouverte à la critique avec le monde universitaire et de la recherche (dans le domaine de la science politique, celui-ci est d'ailleurs souvent concurrencé par la qualité des enquêtes d'un certain nombre de journalistes). Ahmed souligne, par exemple, que « les militaires ont une profusion d'informations factuelles sur le militantisme au Cachemire mais aucune vision analytique des raisons pour lesquels ils pourraient être incapables de le contrôler ». Et il ajoute "Prenez la guerre de 1965. Comment avoir pu imaginer qu'il suffirait d'envoyer 7000 soldats dans la vallée du Cachemire pour provoquer un 
soulèvement? Comment ont-ils pu croire que l'Inde ne réagirait pas et que la Chine interviendrait en leur faveur? Dans ces conditions, pourquoi s'être engagé dans cette guerre désastreuse? ». Or ce bilan peut, dans une large mesure, s'appliquer à la crise de Kargil. L'inertie de l'armée s'est également manifestée dans le choix d'impliquer et de mettre en avant des mouvements islamistes armés dans la crise de Kargil. Elle a reproduit en effet ici un modèle éprouvé, avec succès, en Afghanistan : conceptualiser la lutte comme un «jihad», une "guerre sainte » contre l'oppresseur infidèle indien, comme cela avait si bien marché contre l'Union soviétique. Rappelons d'ailleurs que le lieutenant-général Aziz, un des principaux stratèges de Kargil, a été en charge du bureau afghan de l'ISI (principal service de renseignement de l'armée). L'ISI est en effet le principal pilier de ce volet de la stratégie militaire. Or, selon nombre d'observateurs, cette institution, si elle ne peut être totalement dissociée de l'armée (elle est dirigée par un général de haut rang), possède sa propre politique cachemirie (et sa "Kashmir cell »). Elle a surtout un intérêt propre dans le prolongement du conflit avec l'Inde. « Le budget, la main d'œuvre et le poids de l'ISI sont mieux défendus lorsqu'il y a un ennemi clairement identifiable" précise Ahmed Rashid, bon connaisseur de la politique afghane de l'ISI $^{32}$. Son influence sur l'armée, comme sur les gouvernements civils, provient de ce qu'elle maîtrise deux instruments clés : l'information et la main d'œuvre nécessaires pour toute opération au Cachemire indien. L'armée, et donc ici plus précisément l'ISI, a fait « ce qu'elle sait faire » à un degré de reproduction remarquable. La nature des mouvements que l'ISI aide au Cachemire a, effectivement, radicalement changé dans les années $90:$ du séculariste Jammu\&Kashmir Liberation Front (JKLF) aux groupes islamistes non seulement les plus radicaux mais aussi selon une évolution qui a suivi leur politique en Afghanistan (du Hizb Ul-Mujahidin, proche du Hezb-i Islami afghan, au Harkat Ul-Mujahidin très lié aux Taleban). Le tournant serait intervenu vers 1993 lorsque l'ancien COAS, le général Mirza Aslam Beg, a décidé de reproduire le modèle afghan et de promouvoir un esprit de lutte inspiré par la motivation religieuse, afin aussi d'attirer les soutiens des pays islamiques. Cette politique aurait même fait l'objet d'un haut degré d'organisation: l'ISI aurait ainsi établi un centre de coordination qui, à partir de son quartier général à Peshawar (à quelques kilomètres de l'Afghanistan), entraînait les combattants et assurait le transfert des armes afghanes vers le Cachemire ${ }^{33}$. Un ancien directeur de l'ISI attribue cette évolution plus aux affinités personnelles de l'un de ses prédécesseurs, Hamid Gul, avec les mouvements islamistes qu'à une stratégie planifiée au niveau des chefs d'état-major. Mais il ne dément pas qu'effectivement à un moment donné le choix ait été fait de promouvoir des groupes "jihadistes». L'armée a donc répliqué, dans l'opération de Kargil, des "configurations régulières de comportement" selon les termes-mêmes de Graham Allison : $\mathrm{a} /$ accorder la priorité à l'offensive contre toute autre option b/ privilégier jusqu'au bout la logique strictement militaire au détriment du volet diplomatique c/ impliquer des mouvements islamistes à l'instar de la politique menée en Afghanistan. Mais cette inertie, en se heurtant à une nouvelle donne dont l'armée n'avait peut-être pas mesuré tout l'impact en politique étrangère - l'autonomisation croissante du premier ministre - allait créer les pires effets du point de vue de la gestion de la crise et de la crédibilité internationale du Pakistan. Les limites du modèle du jeu politique : la logique du cavalier seul du premier ministre C'est l'absence de marchandage bureaucratique, répétons-le, qui explique les deux principales incohérences de la crise de Kargil : l'engagement d'une offensive majeure en pleine reprise du dialogue avec l'Inde et le volte-face du premier ministre à Washington. Le fiasco de Kargil serait-il 
pour autant à mettre sur le compte d'un premier ministre victime de l'armée ? Cette question a fait couler beaucoup d'encre au Pakistan. Le simple fait qu'elle ait été soulevée montre bien la difficulté à attribuer clairement les responsabilités décisionnelles au sein de l'Etat pakistanais et par là-même l'ampleur de la crise de gouvernabilité que connaissait le pays quelques mois avant le coup d'Etat du 12 octobre 1999. Nawaz Sharif a largement joué sur la désinformation en tentant de convaincre qu'il avait été mis devant le fait accompli par une armée revancharde. Que de nombreux journalistes l'aient cru ne surprend pas. Il y avait eu des précédents. En mars-mai 1990 déjà, l'Inde et le Pakistan avaient été au bord d'une guerre ouverte qui n'avait été évitée in extremis qu'après une médiation américaine. Or, le premier ministre de l'époque, Benazir Bhutto, avait alors été court-circuité par le COAS général Aslam Beg, seul interlocuteur des Etats-Unis tout au long de la crise ${ }^{34}$. Mais neuf ans après (et neuf ans de gouvernement civil), Kargil offre une image bien différente du rapport de force civils-militaires. Si Nawaz Sharif a été tributaire de l'armée et de l'ISI (de leur perception, de leur savoir-faire, de leur monopole sur les affaires de défense) une fois l'opération engagée, il a bien été informé de l'intervention de Kargil et ce dès janvier 1999, et l'a approuvée ${ }^{35}$. D'autre part, lors de son deuxième gouvernement, l'opération de Kargil avait été proposée à deux reprises à Benazir Bhutto par les militaires qui s'étaient inclinés devant son refus (elle l'estimait intenable diplomatiquement et économiquement) ${ }^{36}$. On ne voit donc pas pourquoi cette fois-ci l'armée aurait pris le risque de procéder autrement. La réalité a plutôt été un renversement, sans précédent en dix ans de démocratie, du rapport de force entre le gouvernement et l'armée. Le premier ministre semble en effet avoir fait le pari, risqué (puisqu'il allait lui coûter son poste), de conduire sa propre politique étrangère en cherchant lui-même à instrumentaliser l'armée, bref de mener double-jeu. Seule une analyse de ce processus inédit - et donc de la conjoncture de politique intérieure tout à fait particulière qui a précédé la crise de Kargil - explique l'ampleur des ratés du processus décisionnel. Depuis un an déjà avant la crise de Kargil en effet, les relations entre l'armée et le premier ministre - dont la concorde, basée sur la quasi-sacralité de l'institution militaire, est la seule garantie de stabilité dans le système de démocratie surveillé que connaît le Pakistan - s'étaient considérablement dégradées en raison d'une série de mesures prises par Nawaz Sharif pour "nettoyer" l'espace de dissensions qui s'élargissait autour de lui. Il y eut tout d'abord, en 1998, un premier amendement constitutionnel ôtant au Président de la République le pouvoir de démettre le gouvernement - et donc à l'armée tout levier de pression sur le premier ministre - puis un second, interdisant aux députés de voter contre leur parti sous peine d'en être exclus. Vint ensuite la démission imposée par Sharif du président de la République Farooq Leghari et les assauts contre la Cour Suprême. Puis le premier ministre "s'attaqua » à l'institution militaire elle-même : en octobre 1998, il obtint la démission du chef d'état-major de la Marine puis, fait sans précédent, du militaire le plus haut placé, le COAS Jehangir Karamat, pour avoir osé critiquer publiquement les performances du gouvernement. Même après la nomination du nouveau COAS Musharraf, qu'il estimait lui être acquis, il ne put faire taire les critiques au sein de l'armée quant à son refus répété de réunir le DCC pour discuter aussi bien de la politique vis-à-vis de l'Inde que de l'Afghanistan. Il démontrait ainsi sa ténacité à prolonger le bras de fer engagé avec l'armée dans la conduite même de la politique étrangère. Cet aspect allait se révéler déterminant. Une évolution importante s'était en effet produite dans la gestion de la politique étrangère du Pakistan: l'amorce, à 
l'initiative du premier ministre, d'une politique régionale autonome vis-à-vis de l'armée et dont l'axe central était la normalisation des relations avec l'Inde. Sharif a joué un rôle de premier plan dans la reprise du dialogue avec le premier ministre indien I. K. Gujral en 1996-97. Il est le premier politicien punjabi à avoir affirmé publiquement en 1997 être prêt à reconsidérer la position jusque là intangible du Pakistan à l'égard de l'Inde. Si ces gestes ne se sont pas traduits par des décisions concrètes quant à l'avenir du Cachemire, les relations entre les deux pays s'étaient réellement améliorées dans les mois qui ont précédé l'intervention de Kargil. Un nouveau régime des visas pour les Pakistanais se rendant en Inde, un service de bus régulier entre Lahore et Delhi, des échanges de prisonniers civils et des rencontres de délégations commerciales et culturelles dans l'un et l'autre pays avaient été instaurés. Surtout, Nawaz Sharif a, semble-t-il, pris seul et contre l'avis de l'armée, la décision d'inviter le premier ministre indien à Lahore en février 1999. L'armée était non seulement consciente de l'incohérence d'un tel geste avec les préparatifs en cours de l'intervention à Kargil (que Sharif avait donc approuvée) mais aussi, comme nous l'avons vu, profondément hostile à toute discussion avec l'Inde tant que la question du Cachemire n'était pas directement abordée. Selon certaines sources, il y aurait même eu des négociations informelles sans précédent en plein préparatifs de l'opération de Kargil ${ }^{37}$. Pour la première fois, toutes les options auraient été envisagées, y compris la possibilité de transformer la $\mathrm{LoC}$ en frontière internationale. Les deux parties se seraient aussi engagées à atténuer l'intensité de leur propagande, à discuter ouvertement de la question des infiltrations transfrontalières et à tenir secrètes l'avancée des négociations qui devaient véritablement s'engager en octobre 1999 au niveau des secrétaires d'Etat aux affaires étrangères. Il semblerait même que le général Musharraf ait été très agacé de cette "diplomatie secrète", dont le contenu réel lui était inconnu et aurait demandé à plusieurs reprises au premier ministre de l'en informer, sans succès. Comment expliquer l'attitude incohérente du premier ministre consistant à approuver une offensive militaire qu'il aurait pu refuser à l'instar de son prédécesseur, tout en engageant parallèlement des négociations secrètes sur le Cachemire ? Comment comprendre également la "trahison » de Sharif à Washington, parti en s'engageant semble-t-il auprès des militaires à obtenir des concessions diplomatiques (ou tout du moins que la LoC ne soit pas mentionnée dans la déclaration pakistano-américaine) et revenant avec un accord de retraite unilatérale ${ }^{38}$ ? L'hypothèse la plus plausible (difficilement vérifiable vu l'impossibilité de recueillir, depuis le coup d'Etat, des témoignages de proches de Sharif mais cohérente avec sa pratique politique) suggère qu'il a voulu faire d'une pierre trois coups. Avoir «le beurre » - se présenter comme un "héros » si l'opération de Kargil réussissait et être en position de force dans les futures négociations sur un réajustement de la LoC "l'argent du beurre" - pacifier les relations avec l'Inde pour promouvoir les exportations, principalement sucrières, de son propre groupe industriel Ittefaq ${ }^{39}$ - et «le sourire de la crémière "- préserver le soutien des Etats-Unis et sauvegarder l'image d'un pacifiste victime des militaires si les choses tournaient mal. Cette hypothèse est d'autant plus plausible qu'un des aspects les plus troublants de la politique pakistanaise au cours de la crise de Kargil fut le fait que les officiels du ministère des affaires étrangères aient été tenus à l'écart et les ambassades à l'étranger, ni informées, ni consultées. Un diplomate en poste à Washington a ainsi déclaré : « Le gouvernement ne nous a pas demandé un seul compte-rendu depuis que la crise a commencé. Ils n'ont indiqué aucune stratégie à suivre ni ligne d'action. Nous 
travaillons dans le vide $\aleph^{40}$. Or, cette situation n'a pas tant été le fait d'une désorganisation administrative que de la volonté expresse du premier ministre (qui aurait d'ailleurs demandé à l'armée d'en faire de même). Se poursuivait en fait dans le champ de la politique étrangère, la conception personnelle et patrimonialiste du pouvoir qui caractérisait le deuxième gouvernement Sharif (1997-1999). Le premier ministre et son entourage étaient devenus en effet en 1999 une clique totalement autonome des parlementaires (il ne légiférait plus que par voie d'ordonnances) comme du gouvernement (transformé en chambre d'enregistrement des décisions qu'il prenait avec quelques-uns de ses proches ${ }^{41}$. Nawaz Sharif dirigeait le pays de façon quasiclanique, à tel point que la presse populaire ne le désignait plus que sous le sobriquet de « empereur Shah Jahan II » et son entourage par la formule fort explicite de «clan de Raiwind» (ville où se trouve la propriété familiale). Sharif prenait ses décisions après consultation d'une petite coterie composée de membres de sa famille et de ses amis. L'emprise de sa famille, et tout particulièrement celle de son père (caricaturée dans la presse sous la forme d'un Nawaz Sharif enfant s'exclamant « et maintenant Papa qu'est-ce qu'on fait?») et de son frère Shahbaz Sharif, puissant Chief Minister (premier ministre provincial) du Punjab, s'est illustré à de nombreuses reprises. Semble d'autre part n'avoir été informés de l'opération de Kargil que le chef de l'ISI, alors un ami de longue date et au sein du gouvernement, les ministres ayant précédemment occupé des fonctions au sein du conglomérat industriel familial Ittefaq (l'avocat de la famille devenu ministre de la justice, l'ancien partenaire commercial doté de la présidence de la puissante Commission anti-corruption, le comptable d'Ittefaq auquel fut donné le poste de ministre du commerce et des finances et enfin un vieil ami journaliste promu ministre de l'information). Nawaz Sharif en étant donc venu à gérer le pays comme son groupe industriel, dans l'ignorance aussi bien de son propre parti, la Ligue Musulmane (qui le lui rendra bien par l'absence générale de soutien après son renversement par l'armée) que du gouvernement. Cette gestion personnalisée du pouvoir qui a conduit à l'exclusion de l'administration des affaires étrangères tant en amont (décision) qu'en aval (accord de Washington) de la crise de Kargil, couplée à l'absence de stratégie diplomatique de l'armée, a eu un impact déterminant. N'ayant aucun discours à opposer à celui qui le ciblait comme saboteur de paix, «Etat paria » et " fondamentaliste », le Pakistan ne pouvait que perdre dans la guerre discursive face à l'Inde. Il a été perçu comme l'unique agresseur dont la seule motivation était de changer la LoC (alors qu'il visait avant tout, rappelons-le, à répondre à l'intensification de la pression indienne accrue sur la portion méridionale de celle-ci). Pour comble d'ironie, c'est le Pakistan et non l'Inde qui fut attaqué pour non-respect des résolutions du Conseil de sécurité, lors du débat au House Foreign Relations Committee américain (par 22 voix contre 5). Le Pakistan a ainsi détruit en deux mois, par l'absence tout bonnement d'une "politique étrangère", ce qu'il tentait d'obtenir et avec de bons arguments, depuis des décennies : une sympathie de l'opinion publique internationale à l'égard de son approche légaliste du dossier du Cachemire. Plus grave, a été ridiculisée auprès de ses partenaires étrangers l'une des seules administrations pakistanaises, les affaires étrangères, qui fait preuve d'une approche souple en matière de relations avec l'Inde et donc, l'une des seules audibles par les partenaires américains et européens. Certains hauts fonctionnaires des affaires étrangères considèrent en effet que la revendication d'un plébiscite basé sur l'appartenance religieuse au Cachemire, de même que son soutien aux mouvements jihadistes (et son aide aux Talebans afghans) sapent la crédibilité de la défense pakistanaise sur ce dossier. D'autres sont aussi prêts à 
envisager des solutions jusque-là taboues au Pakistan, telle la tenue d'un plébiscite limité à l'Azad Cachemire et à la Vallée de Srinagar (excluant donc le Jammu, le Ladakh et les Territoires du Nord pakistanais), sur une base de districts, et incluant la troisième option demandée par le JKLF (l'indépendance). Les incohérences de la gestion de la crise de Kargil relèvent donc principalement de la rupture de l'équilibre sur lequel reposait toute la politique étrangère depuis la création même de l'Etat: la primauté incontestable et sans partage de l'armée sur ce secteur d'activité. La situation nouvelle que connaissait le système politique pakistanais, celle d'un premier ministre qui, agissant avec une autonomie et un autoritarisme inégalés depuis dix ans, devenait de plus en plus ingérable pour les militaires, relativise donc la portée de l'hypothèse de complémentarité entre les trois modèles sur laquelle est fondée l'analyse d'Allison. Les approches complémentaires : le « jeu à deux niveaux » et la dimension émotionnelle de la politique étrangère L'application des modèles d'Allison à la crise de Kargil, même affinés en tenant compte de leur hiérarchie interne, ne donne toutefois qu'une vision encore partielle des dysfonctionnements du processus décisionnel pakistanais. Il importe de considérer également les tensions internes à chaque bureaucratie (et non exclusivement entre elles) ainsi que le poids des affects (et non des seules perceptions), variables dont Allison sous-estime l'importance. Le modèle du "jeu à deux niveaux " Les «voix multiples» du Pakistan sur le dossier du Cachemire ne sont pas juste le résultat d'une lutte forcenée entre des groupes aux agendas conflictuels (armée et ISI, premier ministre et son entourage proche, affaires étrangères). En fait, les options des décideurs ne sont pas toujours clairement identifiables : c'est au sein de chaque groupe que les erreurs d'appréciation, les contradictions, voire la schizophrénie, surprennent. Il serait donc risqué de ne retenir que les rapports de force institutionnels et d'occulter les incohérences émanant d'une seule et même bureaucratie. Celles-ci peuvent se comprendre à l'aide de la métaphore, simple mais efficace, du " jeux à deux niveaux » (two-level game) élaboré par Robert Putnam. Le politiste remarque, à juste titre, que si Allison souligne bien que « le modèle de la politique bureaucratique dirige l'attention vers les jeux intra-nationaux dont les chevauchements constituent véritablement les relations internationales ", il reste allusif sur la nature de ces " chevauchements $»^{42}$. Il s'agit donc d'élucider ce que Putnam appelle les «interactions paradoxales ", c'est-àdire les effets de calculs potentiellement contradictoires concernant les avantages attendus de l'action au niveau national et les gains anticipés au niveau international. La tension entre "volonté de maximiser sa propre capacité à satisfaire des groupes de pression internes (dans le cas qui nous occupe les mouvements islamistes principalement) tout en minimisant les conséquences adverses dans ses relations avec ses partenaires étrangers » (ici surtout les Etats-Unis et les organisations politiques cachemiries indiennes) a caractérisé les évaluations de l'armée comme du premier ministre pendant la crise Kargil. Les calculs contradictoires de l'armée Dans le choix de l'offensive contre l'Inde, l'armée poursuivait comme premier objectif interne de relégitimer l'institution militaire. L'opération de Kargil - dont elle anticipait une issue victorieuse - lui aurait permis en effet de redorer son blason. Rappelons qu'elle intervenait dans un contexte marqué par une forte déstabilisation de l'institution militaire. Sous le choc depuis la démission forcée de son plus haut dirigeant et la valse de changement aux postes de commande depuis l'automne 98, elle subissait également les assauts tout azimut du premier ministre. Celui-ci n'avait pas seulement cherché à désolidariser de l'état-major de l'armée de terre l'ISI mais aussi les neuf commandants de corps d'armée (tous membres de l'armée de terre et acteurs décisifs, avec l'ISI, dans 
la mise en œuvre de la politique étrangère). Enfin, il tentait de détruire le capital de sympathie dont dispose traditionnellement l'armée au Pakistan ${ }^{43}$ en laissant ses ministres la critiquer ouvertement dans la presse. La charge commençait à porter ses fruits si l'on en croit ce commentaire inattendu d'un jeune punjabi d'origine modeste : «l'armée a perdu toutes les guerres contre l'Inde, elle ne se bat plus depuis vingt-sept ans, elle reste dans les casernes et avale notre budget. Les mujahidin, eux, ils se battent vraiment contre l'Inde ! $»^{44}$. Pour certains jeunes tentés par l'action militaire, il est en effet devenu plus valorisant de rejoindre leurs rangs que ceux de l'armée. Il s'agissait donc également pour l'armée de contrer l'influence croissante des mouvements jihadistes ${ }^{45}$ et de donner des gages aux différents partis islamistes pakistanais. Si l'on évalue la force de ces mouvements à peut-être 300 ou 400000 hommes $^{46}$ - alors que l'armée régulière en compte 500000 - l'inquiétude de cette dernière est fort compréhensible. En outre, les groupes qui ont participé à l'opération de Kargil sont tous, peu ou prou, soit des émanations des partis d'opposition islamistes sunnites, soit intimement liés à eux : le Hizb Ul-Mujahidin et Al-Badr Mujahidin au Jama'at-i Islami (JI), le Harkat-Ul Mujahidin au Jamiat-e Ulama-e Islam (JUI) et le Lashkar-e Taïba au Jamiat-e Ahl-e Hadith (JUAH) ${ }^{47}$. C'est d'ailleurs la raison pour laquelle l'aide de l'ISI à ces groupes, dont elle a besoin pour mener les opérations de destabilisation au Cachemire, a toujours évolué en fonction de la croissance de leur ambition politique nationale et $d u$ principe $d u$ "diviser pour mieux régner» (en jouant sur leur concurrence idéologique). Il ne faudrait toutefois pas surestimer le contrôle que les services secrets exercent sur eux : il ne s'agit pas de mercenaires mais de militants endoctrinés et motivés auxquels l'Etat sous-traite une partie de sa politique régionale. Un groupe comme le Harkat Ul-Mujahidin par exemple échappe partiellement au contrôle de l'ISI et parce qu'il a été actif au Cachemire (et parallèlement en Afghanistan) avec sa bénédiction, il ne peut directement le confronter ${ }^{48}$. En impliquant donc les groupes jihadistes dans une opération conventionnelle telle que Kargil et en leur attribuant dans ses déclarations officielles l'entier succès de l'intervention (pour masquer sa propre présence), l'armée estimait contribuer à limiter leur militantisme interne qu'elle perçoit (à tort) comme inversement proportionnel à leur engagement externe. Elle leur rendait également un fier service dont ces groupes pourraient être reconnaissants. Depuis plus d'un an en effet, les mujahidin étaient confrontés à des difficultés opérationnelles croissantes. Après avoir été en partie repoussés de la Vallée de Srinagar, ils se sont repliés vers le Jammu mais les Rashtarya Rifles de l'armée indienne les ont obligés en 1997 à un autre déplacement tactique vers la zone de Kargil (à majorité chiite et pourtant indifférente à l'Azadi movement) ${ }^{49}$. Les calculs internationaux quant à eux, n'étaient pas exclusivement stratégiques (contraindre donc l'Inde à mettre un terme aux tensions le long de la LoC à l'est du Cachemire). Il s'agissait aussi de remettre au cœur de l'agenda des membres du Conseil de sécurité un dossier dont ils ont la responsabilité depuis cinquante ans mais qu'ils se refusent à aborder dans les termes des résolutions de 1948, 1949 et 1957. Il devenait également impératif de regagner la confiance des mouvements cachemiris indiens à l'égard du Pakistan, d'envoyer des signaux positifs à des militants dont la lutte commençait à s'épuiser depuis le milieu des années 90. En effet, comme le notait dès 1996 un des rares chercheurs à s'être rendu au Jammu et Cachemire indien, "l'Etat indien, avec ses ressources policières et militaires considérables, montre maintenant une plus grande résistance et endurance que le mouvement de guérilla cachemiri /.../ clairement sur le déclin depuis 1994 aussi bien dans les zones rurales qu'urbaines ${ }^{50}$. Si l'on en croit les 
chiffres (les seuls disponibles) de l'armée indienne: 1351 membres des troupes indiennes ont été tués au Cachemire indien entre 1990 et $1999^{51}$, tandis que les militants armés cachemiris auraient perdu peut-être 30 fois plus de militants durant la même période ${ }^{52}$. Or, ces calculs internes et internationaux (relégitimer l'armée, contenir les ambitions des mouvements jihadistes sur la scène interne, forcer l'Inde au chantage tactique, rassurer les mouvements cachemiris et attirer l'attention internationale sur le Cachemire) étaient intrinsèquement contradictoires. Les faire avancer au moyen d'une guerre altérant le statu quo frontalier, tout d'abord, était inadmissible pour les principaux alliés du Pakistan, aussi bien les Etats-Unis que la Chine. Le désaveu se lit en effet clairement à travers les lignes de la déclaration américano-pakistanaise du 4 juillet 1999 qui a finalement contribué à « sanctifier » la LoC et à réaffirmer la position indienne que seule une solution bilatérale (et non multilatérale) était acceptable ${ }^{53}$. Il n'est fait nulle part mention des résolutions du Conseil de sécurité et l'hypothèque d'une pression internationale accrue en vue d'une transformation de cette ligne de partage, au moins reconnue jusque-là comme " disputée », en frontière internationale est ainsi plus lourde que jamais. La Chine, particulièrement sensible à toute modification des statu quo territoriaux, a de même appelé le Pakistan à respecter la LoC et ce, alors même que Nawaz Sharif se trouvait à Pékin. Si la diplomatie et les groupes de pression indiens aux Etats-Unis ont pu sans trop de crainte mener une vaste campagne médiatique sur le thème du Pakistan «Etat terroriste », c'est aussi parce que l'armée a proclamé haut et fort que les «mujahidin », soutenus juste "moralement et politiquement » par le Pakistan, menaient l'opération. Si depuis lors, l'armée a changé sa stratégie de communication (revendiquant la mort de ses propres « martyrs » lors de la bataille de Kargil), elle n'en a pas moins commis alors, selon le jugement Maleeha Lodhi dont les analyses se révèlent là encore fort éclairantes, « un véritable désastre de relations publiques $»^{54}$. En effet, l'implication marginale des mujahidin (postés en contrebas des sommets) et celle, directe, de l'armée s'est rapidement révélé être un secret de polichinelle. Avant même la cessation des hostilités (dont les modalités, nous l'avons souligné, le rendait manifeste), le rôle déterminant de la Northern Light Infantry (force paramilitaire encadrée par des officiers de l'armée de terre et depuis intégrée à l'armée régulière) qui a perdu près de 300 membres dans ce conflit, a été publiquement souligné par des analystes de défense liés à l'establishment militaire, d'anciens directeurs de l'ISI et même un ancien $\mathrm{COAS}^{55}$. Ces incohérences ont mis une fois de plus l'armée en porte-à-faux avec son "allié " américain qui lui intime de mettre un terme à l'aide matérielle apportée aux mouvements de guérilla islamistes et tout particulièrement au Harakatul Ansar dont les militants ont été visés lors des bombardements américains d'août 1998 contre les camps d'Oussama Ben Laden en Afghanistan où ils s'entraînaient ${ }^{56}$. Concernant les signaux positifs qu'il s'agissait d'envoyer aux grands mouvements politiques cachemiris basés en Inde, le Pakistan a obtenu l'inverse. Il s'est tout d'abord aliéné la All Parties Hurriyat Conference (APHC), le prernier organe politique de représentation des différents partis et mouvements de lutte cachemiris indiens $\mathrm{s}^{57}$. Les représentants de la APHC au Pakistan ont ainsi durement ressenti que le "véritable leadership cachemiri ", à savoir eux-mêmes, aient d'abord été exclus de toute consultation pendant la crise, puis finalement « trahis " par l'accord de Washington ${ }^{58}$. A une question portant sur ce que le Pakistan pouvait encore faire pour les Cachemiris, Abdul Ghani Lone, une des figures de proue du «azadi movement », a sèchement rétorqué : « qu'il nous fiche la paix ! " $^{59}$. Cette désaffection de la APHC n'est pas sans importance pour l'Etat pakistanais qui continue de proclamer 
qu'il est le seul organe représentatif des Cachemiris. Il reste effectivement le groupe dont le lobbying international est le plus performant et le groupe le plus crédible ${ }^{60}$. En dépit des divisions qui le traversent depuis six ans, il parvient en effet à survivre en raison de la force de l'attachement de tous ses membres au plus petit dénominateur commun qu'est le référendum d'autodétermination. Le Pakistan s'est aussi mis à dos le Jammu-Kashmir Liberation Front (JKLF), mouvement traditionnellement méfiant à l'égard du Pakistan puisqu'il est hostile au rattachement au Pakistan et favorable à l'indépendance du Cachemire. Le JKLF, qui a son propre mouvement armé, n'a pas été sollicité pour participer à l'opération de Kargil. Or, il garde et ce bien qu'il soit lui aussi divisé, une réelle aura. L'option de l'indépendance gagne en effet en popularité aussi bien auprès des Cachemiris indiens qu'auprès des Pakistanais ${ }^{61}$. Même un activiste du Hizb Ul-Mujahidin au Cachemire indien reconnaissait en 1996: «Je suis un supporter ardent du rattachement au Pakistan mais je crois que $80 \%$ des gens soutiennent la notion d'indépendance $»^{62}$. Le Pakistan, en soutenant des groupes islamistes dans une région marquée par l'islam soufi et syncrétique que l'idéologie rigoriste de ces groupes précisément condamne, favorise lui-même cette tendance qui le dessert ${ }^{63}$. Le fossé avec la population cachemirie se creuse d'autant que les mouvements d'inspiration islamistes soutenus par le Pakistan sont largement perçus comme des "étrangers". Tous leurs dirigeants sans exception sont des vétérans du « jihad » afghan et la plupart recrute leurs militants au Pakistan, au Punjab et dans la North West Frontier Province ; leur base sociale au Cachemire indien (certes difficile à évaluer) semble faible. Enfin, même le calcul interne s'est révélé erroné. En cherchant à contrer l'influence des groupes jihadistes sur la scène politique pakistanaise, l'armée a finalement contribué à sa progression. L'opération de Kargil a en réalité été un formidable "coup de pub » pour ces mouvements. Le Lashkar-e Taïba, par exemple, rassemblait 100000 participants lors de sa convention annuelle de 1998, il en réunissait 200000 en novembre 1999. Au lendemain de l'opération, vue l'explosion de la «demande », ces mouvements auraient même dû établir des listes d'attente de militants prêts à partir au «jihad» au Cachemire et de nouveaux camps d'entraînement ont été ouverts, principalement dans les Northern Areas ${ }^{64}$. Or, tous ces groupes, qu'ils le proclament ouvertement ou par l'intermédiaire des partis auxquels ils sont liés, attendent des dividendes internes de leurs interventions en Inde et des "sacrifices " qu'ils ont endurés pour la cause du Pakistan. On a ainsi entendu Hafiz Muhammad Sayid, leader du Lashkar-e Taïba, proclamer au lendemain du coup d'Etat du 12 octobre 99 et de la suspension de la Constitution: "Je présente au Général Musharraf la constitution d'Allah $»^{65}$. Ces ambitions seront tout aussi difficiles à gérer par les militaires qu'elles le furent pour le gouvernement civil. Les objectifs paradoxaux du premier ministre Le premier ministre a également retenu des options contradictoires tout au long de la crise de Kargil qui s'expliquent par le "jeu à deux niveaux", tant les tensions entre calculs internes et internationaux étaient fortes. Approuver l'opération et tenir un discours va-t-en-guerre tout en dialoguant parallèlement avec l'Inde pour finalement négocier un ordre de retraite unilatéral, ces incohérences s'inscrivent en réalité dans le cadre plus large d'une politique cachemirie marquée depuis le début du gouvernement Sharif du sceau de la schizophrénie. Ainsi, alors que s'engageait à l'automne 1998 une nouvelle série de discussions bilatérales sur différents dossiers de contentieux (paix et sécurité, terrorisme et trafic de drogue, glacier du Siachen, projet du barrage de Wullar, délimitation des eaux territoriales, coopération économique et commerciale, etc.), le gouvernement ne cessait de répéter que ces « discussions » n'auraient jamais lieu si la 
question du Cachemire n'était pas réglée au préalable. Or, elles eurent bien lieu, le gouvernement se satisfaisant d'une vague promesse indienne de "discuter du Cachemire " (la stratégie indienne de "délier» le Cachemire des autres dossiers conflictuels avait réussi). Au lieu d'inonder la presse de déclarations sur l'inévitable échec des pourparlers, pourquoi ne pas avoir alors mis en place une politique de communication cherchant à convaincre de la nécessité du dialogue ? De même, pendant Kargil, pourquoi ne pas avoir assumé dès le départ les différences de vues avec l'armée ? C'est que le calcul de politique étrangère consistant à préserver le soutien des Etats-Unis en montrant sa bonne volonté à négocier avec l'Inde rentrait en contradiction avec un impératif de politique intérieure fort : aucun gouvernement au Pakistan n'est prêt à courir le risque d'être perçu comme étant « lâche » sur la question du Cachemire. Ceci est vrai pour Sharif comme pour Benazir Bhutto. Tous deux ont été, par exemple, obligés de se rétracter en invoquant des propos déformés par le journaliste après avoir envisagé la possibilité de l'indépendance pour le Cachemire. Evoquer même l'option d'un plébiscite ne couvrant pas la totalité du Cachemire dans les limites de l'Etat princier serait un "suicide politique». La pression interne est en effet considérable. Le Cachemire est tout d'abord partie intégrante de la politique de vendetta, de rivalité et de revanche entre les deux grands partis de gouvernement depuis le retour à la démocratie en 1988. En 1991 par exemple, le parti de Sharif, la Pakistan Muslim League, fut obligé d'organiser une grève nationale de soutien à la résistance cachemirie après une intense campagne du Pakistan People's Party (PPP) de Benazir Bhutto dans la presse sur le thème : «Nawaz Sharif a été porté au pouvoir pour transiger sur la question du Cachemire ». De même, lorsqu'un service de bus régulier fut rétabli entre l'Inde et le Pakistan, c'est un autre parti d'opposition, le Jama'at-i Islami qui, sur le thème cette fois de la bonne veille théorie du complot (la " conspiration hindoue ») organisa des manifestations violentes contre la présence de Vajpayee à Lahore. L'opposition à toute normalisation des relations avec l'Inde qui ne mettrait pas un terme à « l'occupation indienne au Cachemire " provient aussi d'autres groupes de pression. Les "kashmiri mohalla» tout d'abord (c'est-à-dire les communautés originaires du Cachemire aujourd'hui indien, ayant immigré juste avant ou après la partition de 1947), évalués à 2 millions de personnes ${ }^{66}$ établies principalement dans les grandes et moyennes villes du nord Punjab. Ceux-ci sont particulièrement bien intégrés dans la société pakistanaise. Ils constituent tout d'abord une véritable "banque de vote» lors des élections législatives d'Azad Cachemire. Ils animent d'importantes ONG, tel que le Kashmir Action Committee, et jouent un rôle non négligeable au sein de puissants "think thank» sur les relations indopakistanaises, tel que le Institute of Regional Studies d'Islamabad. Enfin, les Cachemiris occupent souvent des postes influents dans l'appareil d'Etat où il sont sur-représentés par rapport aux autres groupes ethniques non-punjabis (pashtounes, sindhis et baloutches). Le gouvernement d'Azad Cachemire (2,5 millions d'habitants), formellement autonome bien que totalement dépendant militairement comme financièrement $d u$ gouvernement fédéral pakistanais, agit aussi comme un groupe d'intérêt contraignant. Si son influence est quasi nulle dans l'élaboration de la politique cachemirie, il dispose d'une marge de manœuvre non négligeable car le gouvernement fédéral est tenu, pour démontrer qu'il agit à l'opposé du rival indien dans sa partie du Cachemire, de protéger tous les signes extérieurs de l'autonomie politique. Il compte ainsi comme un des relais important de son lobbying international en faveur du Cachemire. Ses politiciens, fidèles à la devise écrite en lettres immenses sur les collines 
à l'entrée dans la province ( « Le Cachemire appartient au Pakistan ») utilisent toutes les ressources dont ils disposent (aussi bien les médias que le lobbying auprès des officiels étrangers) pour exercer une pression vigilante contre tout compromis au Cachemire (qui limiterait leur statut privilégié) . Enfin, le gouvernement doit compter avec les opinions, très hostiles à l'Inde, véhiculées dans la presse populaire et tout particulièrement celle de Lahore, poumon médiatique du pays. Le deuxième quotidien national en ourdou, Nawaz-e-Waqt, par exemple, bien que soutien traditionnel de Sharif, n'hésita pas à lancer une violente campagne contre le processus de normalisation qu'il engageait à l'automne 1998. Gérant lui-même un «Kashmir Relief Fund " pour aider les réfugiés cachemiris et les mouvements jihadistes, ces colonnes sont nourries de récits des souffrances des premiers et des exploits des seconds. D'une façon générale, en trois années de lecture de la presse pakistanaise, nous n'avons pas souvenir d'une seule journée où le drame du Cachemire n'ait pas été abondamment évoqué. Dans un tel contexte, le gouvernement n'aurait pu compter que sur le soutien des mouvements pacifistes tels que la Human Right Commission (et le Pak-India People's Forum for Peace and Democracy que celui-ci anime). Mais, le premier ministre, dans sa politique autoritariste de muselage de toutes les ONG osant critiquer le degré de corruption et d'atteintes aux droits de l'homme de son régime, a lui-même sapé cette option. Les contradictions qui ont donc caractérisé l'ensemble des décisions prises au cours de la crise de Kargil aussi bien par l'armée (mener une offensive au-delà de la LoC impliquant des groupes jihadistes pour internationaliser le conflit, sans toutefois en apparaître comme l'instigateur) que par le premier ministre (accepter la proposition des militaires puis solliciter l'aide américaine pour leur imposer ensuite un ordre de retraite unilatérale) se comprennent mieux en prenant en considération "l'inhabituelle complexité du jeu à deux niveaux » sur l'enjeu du Cachemire. Sans les contredire, elle affine les hypothèses d'Allison. Comme le souligne Putnam, « des gestes qui sont rationnels pour un joueur à une table /.../ peuvent se révéler impolitiques [impolitic] pour le même joueur à une autre table $»^{67}$. Pour banal que soit ce constat, il n'est en pas moins juste et lourd de conséquences politiques. Décisions internationales et charge affective L'émotionnel, objet résiduel de la science politique ? Objet difficile certes, mais dont on pressent sans cesse l'importance, dans le domaine de la décision internationale tout autant que dans d'autres secteurs de l'activité sociale mieux investis ${ }^{68}$. Allison ne s'y est pas attardé bien que l'attitude du président Kennedy pendant la crise des missiles en donne de multiples signes : sa colère face au sentiment d'avoir été « trahi » par Kroutchev; son abattement lorsqu'il reste seul avec son frère (« c'est vraiment moche n'est-ce pas? Mais /.../ s'ils nous font des coups pareils ici... Il n'y a pas le choix »); le désir omniprésent de reconnaissance internationale des alliés (qui, de l'aveu même du Président, «nous considèrent comme un peu névrosés sur cette question »); son emportement («Puis on y va samedi et on les détruit /.../ Et s'il y en a encore... on les bazarde tous !») alors même que l'option d'une réponse modérée aux Soviétiques semble acquise ${ }^{69}$. En dépit du fait que le sociologue soit souvent mal armé pour opérer les indispensables emprunts à la psychanalyse que l'approche du politique par ses dimensions affectives implique, «projections, idéalisations, dénis de réalité, régressions anxieuses tous ces mécanismes, habituellement confinés dans la sphère du psychisme personnel, sont également repérables dans la vie sociale » comme le rappelle Philippe $\mathrm{Braud}^{70}$. Le politiste force l'envie de tenter ce travail de repérage dans l'analyse décisionnelle en relations internationales. Nous nous limiterons donc ici à quelques pistes de réflexion afin d'éclairer le caractère, soit rigide, soit impulsif, d'un 
certain nombre de décisions prises au cours de la crise de Kargil, crise dont l'enjeu, le Cachemire, comme le contexte, une situation d'urgence, ont favorisé, selon nous, un fort investissement émotionnel. Considérer la politique militaire pakistanaise au Cachemire à la lumière de ses ressorts émotionnels éclaire ce que les variables sécuritaire, organisationnelle et interactionniste (entre la scène politique interne et internationale) laissent dans l'ombre; à savoir, la conviction inébranlable ou bien à l'inverse l'hésitation qui a présidé à certains choix. Comment comprendre la décision hautement risquée d'une agression militaire dans un contexte nucléaire, alors même que toutes les précédentes avaient échoué et l'impossibilité à corriger, ou même à atténuer, au fur et à mesure de la crise, les dysfonctionnements et les incohérences politiques patentes? En observant la façon dont les émotions - «tout état affectif qui s'écarte de ce degré zéro qu'est l'indifférence absolue envers un objet» - non pas "coloreraient les mobiles des acteurs» mais «se situent au cœur des processus de construction des intérêts, des aspirations et des exigences $»^{71}$. On proposera l'hypothèse que deux dynamiques psychosociales majeures ont structuré le comportement des décideurs au cours de la crise de Kargil : l'estime de soi et le déni de réalité. L'estime de soi "Une des guerres les plus absurdes » dans laquelle les deux parties ont une "approche tout aussi rigide qu'émotionnelle $»^{72}$. Ces mots sont ceux d'un ancien commandant du 10ème corps d'armée de l'armée pakistanaise. Portant sur le conflit du Siachen, il pourrait aussi s'appliquer au Cachemire (si le drame humain n'y était pas bien plus poignant). La question du Cachemire n'est en effet pas un simple "problème de politique étrangère ", ni même un conflit de tracé de frontière ou un enjeu stratégique. Elle est avant tout un enjeu symbolique. La passion, la colère ou l'exaltation avec lesquelles elle est évoquée par les décideurs - et plus généralement l'ensemble de nos interlocuteurs toutes catégories sociales confondues ${ }^{73}$ - sont si systématiques qu'elles ne peuvent être anecdotiques. Tout jeune écolier connaît par cœur des phrases telles que «le Cachemire est l'agenda inachevé de la partition de 1947 », « le Cachemire est la veine jugulaire du Pakistan » et en tout premier lieu, « le Pakistan est incomplet sans le Cachemire », célèbre formule de Mohammad Ali Jinnah, le fondateur du « Pakistan » (nom acronyme dont la lettre « $K$ » désigne précisément le «Kashmir », le « P », le « A » et le suffixe "stan » évoquant les autres provinces, le tout signifiant « le pays des purs »). La référence au Cachemire est ainsi indissociable d'un discours sur soi, sur le passé, sur la formation de l'Etat pakistanais né, rappelons-le, dans une guerre avec l'Inde au Cachemire, et sur l'identité nationale. Elle stigmatise le non-achèvement de l'Etat (la LoC de 1972 reste une limite temporaire qui d'ailleurs n'apparaît ni sur les cartes officielles, ni sur les cartes scolaires). La mobilisation en faveur du Cachemire, telle qu'elle est orchestrée par les officiels et les médias, est bien moins le fruit d'un " grand dessein consistant à se tailler une sphère d'influence fondée sur le radicalisme sunnite $»^{74}$ que la justification paradoxale - mais le paradoxe n'est qu'apparent si l'on rappelle la solidarité entre le "war making » et le "state making » qui a jalonné l'évolution des Etats européens ${ }^{75}$ - de la précarité de l'Etat et de la force de la principale institution "garante de son unité », l'armée. La cristallisation émotionnelle autour du Cachemire dans l'affirmation identitaire pakistanaise (qui n'a pas son équivalent à l'égard de l'Afghanistan), ne se comprend qu'en évoquant le contexte dramatique de la partition avec l'Inde de 1947. Le Cachemire réveille - et entretient - le souvenir de l'intensité des luttes politiques entre l'intelligentsia musulmane (réunie au sein de la Muslim League favorable à la création d'un Etat séparé pour les musulmans) et le Congrès, qui ont précédé la partition, ainsi que celui des 
traumatismes de sa mise en œuvre (exodes, pillages, massacres faisant au moins 500 000 morts et une quinzaine de millions de personnes déplacées de part et d'autre ${ }^{76}$. Tout se passe depuis lors comme si le rapport du Pakistan à l'Inde ne pouvait être qu'une relation tout aussi hostile que mimétique et fascinée. L'estime de soi, sur le mode nationaliste, peine à se réaliser hors de la dépréciation, voire de la diabolisation, du voisin indien auquel il s'est en quelque sorte "arraché ». Ce jeu de bascule entre valorisation et stigmatisation, dont Denis-Constant Martin a fort bien démonté les ressorts dans la mobilisation identitaire ${ }^{77}$, est d'autant plus puissant au Pakistan qu'il est également terriblement nécessaire. Dans un pays où seulement $7 \%$ de la population parle la langue nationale officielle, où le pouvoir politique est systématiquement appréhendé en termes d'équilibres interethniques et où le référent unitaire islamique, raison d'être de la création de l'Etat, se fragilise au fur et à mesure de l'intensification des luttes sectaires, le travail de survalorisation de soi par le dénigrement de l'Inde fait office de discours nationaliste. La difficulté du Pakistan à sortir d'un rapport fusionnel et anxieux avec son voisin et le rôle crucial que joue à cet égard le conflit du Cachemire, est particulièrement net dans le traitement dont il fait l'objet à la télévision. La chaîne nationale publique PTV produit ainsi, tous les samedis soir, un feuilleton populaire sur le drame du Cachemire dont le titre, «Lâg » - mot quasiment intraduisible qui signifie "connexion» au sens aussi bien d'«inimitié » et d'«affinité », de "complot " et d'«amour» - symbolise bien la nature toute particulière de cette relation indopakistanaises que l'enjeu du Cachemire alimente (Freud parlerait de "narcissisme des petites différences » ... $)^{78}$. «Lâg " raconte l'histoire d'un petit garçon vivant heureux avec ses parents dans un village des environs de Srinagar jusqu'au jour où les forces de sécurité indiennes mettent sa maison à feu et à sang. Ce type de propagande a un effet psychologique et politique qui passe souvent inaperçu. Le témoignage recueilli auprès d'une fillette de sept ans, spectatrice assidue du feuilleton, mérite ici d'être évoqué. Suite à d'horribles cauchemars où des Indiens envahissaient sa maison et brûlaient ses parents, elle écrivit ce poème pour extérioriser son anxiété : «il y a le feu partout au Cachemire et si vous allez là-bas, c'est le sang de votre famille que vous verrez couler ». Sans bien sûr généraliser, on peut toutefois avancer que toute une génération de jeunes Pakistanais est ainsi socialisée à la haine de l'Inde (tout en maintenant un rapport schizophrénique à elle puisque ses stars musicales et de cinéma sont aussi parmi les plus appréciées dans le pays). Elle grandit également dans une compréhension de la question du Cachemire comme ce qui est justement «l'indénouable », ce qui lie «l'ici » et le «là-bas », obscurcissant plus qu'il ne le clarifie le sentiment national pakistanais. On comprend mieux ainsi pourquoi tout compromis sur le Cachemire nécessiterait un long travail de préparation de l'opinion publique et combien l'absence de telles précautions contribua à la popularité des slogans sur « le traître Sharif » à son retour de Washington. La charge émotionnelle que revêt la question du Cachemire et la façon dont celle-ci est entretenue par le jeu de la mémoire, n'est pas qu'une affaire de médias ou de petite fille. Après avoir avancé toutes sortes de raisons fort rationnelles du point de vue stratégique et politique à l'impossibilité de transiger avec l'Inde, un général de haut rang de l'armée pakistanaise nous a ainsi raconté comment, enfant, il adorait jouer avec ses camarades au "grand libérateur du Cachemire " ${ }^{79}$. L'éclat de rire qui accompagna l'anecdote ne la rend pas moins significative (il a d'ailleurs ajouté « Nous sommes si émotifs sur le Cachemire vous savez!»). Elle suggère, qu'interpellé sur une question politique bien concrète, le décideur suprême peut, lui aussi, être pris dans « ce temps qui ne passe pas " pour reprendre le titre du bel ouvrage que J.-B. Pontalis 
consacra à cet enseignement énigmatique de la psychanalyse : «l'inconscient ignore le temps $»^{80}$. Cette ignorance, si bien sûr elle ne détermine pas mécaniquement l'action, contribue à insuffler à la "construction des intérêts et des aspirations » des logiques "irrationnelles", et ce d'autant plus dans ce moment de tension et d'urgence, luimême négation du temps, qu'est celui de la prise de décision en situation de crise. Sans être en mesure de mener une étude sur la façon dont s'organisent les affects des quatre décideurs centraux impliqués dans la crise de Kargil, trois éléments d'information peuvent être utiles pour éclairer la difficulté de l'armée à modifier sa stratégie dans le cours de la crise, tout comme les décisions erratiques du Premier ministre. Il importe tout d'abord de rappeler que les officiers originaires du nord et centre du Punjab représentent plus de $70 \%$ du corps des officiers ${ }^{81}$, soit de la province qui a le plus souffert de la partition. Les récits de vie d'un certain nombre d'officiers abondent en évocations des divisions et déplacements des membres de leur famille et de souvenirs des visions de massacres dont ils ont alors été témoins. La colère, l'humiliation, est aussi entretenue par la mémoire des défaites face à l'Inde et tout particulièrement celle de 1971. La biographie des principaux protagonistes de l'opération de Kargil fournit ici des éléments intéressants pour évaluer la possibilité d'une intériorisation d'une approche offensive des relations avec l'Inde. Le général Musharraf, dont la famille originaire de Delhi a émigré au Pakistan après la partition quand il était enfant, a été actif, comme membre du Elite Special Services Group dans la guerre de 1965 tout comme dans celle de 1971. Le lieutenant-général Mehmood a eu, comme commandant du corps d'armée de Rawalpindi, la responsabilité de la défense de l'Azad Cachemire et de la protection de la LoC. Enfin, le lieutenant-général Aziz est originaire du Cachemire (il est né à Rawalkot, situé aujourd'hui au Cachemire pakistanais, l'année de la partition). Tout au long de sa carrière, il a aussi occupé des postes clés dans la direction $\mathrm{du}$ conflit du Cachemire. Enfin, la famille de Nawaz Sharif est elle aussi originaire du Cachemire et il semblerait que les pressions exercées par son père, tout aussi réticent que l'armée à sa politique de rapprochement avec l'Inde, n'ont pas été étrangères à la difficulté du premier ministre d'arrêter une décision ferme pendant la crise de Kargil. De ce rapport passionnel avec l'Inde, dont les ressorts sont donc autant le fait de trajectoires historiques qu'individuelles, dérive également une politique étrangère que les décideurs pakistanais ne parviennent pas à penser hors d'un jeu de miroir avec leur voisin. La politique du «donnant-donnant» que visait à établir l'opération de Kargil (un coup au Ladakh contre un coup en Azad Cachemire) s'inscrit dans cette logique. Cette façon d'élaborer la politique indienne de façon strictement réactive est une constante. Le Pakistan a, par exemple, engagé son programme de nucléarisation au lendemain des essais indiens de 1974 et n'a sauté le pas en mai 1998 que quinze jours après la série d'explosions et l'intense "guerre psychologique » indiennes (selon le terme employé par les officiels pakistanais). Les déclarations du ministre de la défense indien sur la primauté de la menace chinoise par rapport à celle posée par le Pakistan (alors que celui-ci baptisait son missile balistique de portée intermédiaire du nom de "Ghauri ", guerrier afghan qui avait tenu tête à l'Inde au XIIème siècle !) ont alimenté un fort sentiment d'humiliation qui convaincra finalement les dirigeants pakistanais de procéder eux aussi à leur «explosion d'estime de soi " (pour reprendre le titre judicieux d'un article indien). Le déni de réalité La fuite dans l'illusion et le déni de réalité peut également produire des effets politiques qui ont une importance non négligeable. Si ses origines (négation défensive face à une réalité perturbante, isolement des décideurs, entre autres) sont plus faciles à aborder que ses effets concrets 
sur la prise de décision, elle n'en est pas moins repérable au cours de la crise de Kargil. Elle permet de comprendre la résistance de l'armée à modifier sa stratégie au fur et à mesure que la guerre se perdait sur le front diplomatique et celle du Premier ministre à anticiper les réactions de l'armée à la reddition de Washington. Du début jusqu'à la fin de la crise, le porte-parole de l'armée n'a cessé d'affirmer que Kargil était une brillante opération : l'Inde avait été prise par surprise et obligée de procéder au déplacement de troupes le plus important depuis 1971 (ainsi que d'engager l'aviation). Mais cette "réussite opérationnelle» n'avait, de l'avis de Maleeha Lodhi, aucun sens si elle ne permettait pas d'obtenir des gains concrets, gains qui ne peuvent qu'être négociés sur un plan diplomatique. C'est également le sens de la critique, placée en exergue, du général Karamat: "et maintenant que nous occupons les hauteurs des sommets de Kargil et Drass, que faisons-nous?». L'absence d'options de sortie de crise, soulignée par les deux observateurs, a donc été la manifestation la plus visible du déni de réalité. Il semblerait que cette réaction soit à mettre sur le compte de « la présomption clé de toute l'opération: l'Inde s'inclinera devant cette capture tout comme les Pakistanais ont été obligés d'avaler la prise des pics du Siachen par l'Inde en $1984 »^{82}$. En lançant cette opération, en coupant, sur l'axe Srinagar-Leh, la route d'approvisionnement des troupes indiennes stationnées en contrebas du glacier de Siachen, les militaires estimaient en effet que l'Inde serait condamnée à plier. Certains observateurs avancent que le lieutenant-général Aziz était convaincu que Kargil, opération coûteuse aussi bien humainement que financièrement pour l'Inde, l'obligerait de facto à négocier rapidement. Et quand bien même elle contre-attaquerait, l'armée pensait pouvoir gagner ce qui ne pouvait être qu'une guerre limitée. L'Inde aurait concentré trop de troupes au nord du Ladakh pour pouvoir riposter le long de la frontière internationale et serait tenue par le chantage nucléaire virtuel. Dès lors, avant même l'imposition d'un cessez-le-feu, les militaires pakistanais auraient eu suffisamment de temps pour regagner les portions de territoires perdues en 72 (et donc de revenir au tracé antérieur de la ligne de cessez-le-feu). En d'autres termes, se révélait une des logiques centrales du mécanisme de l'illusion: le "wishfull thinking ", ou prendre ses désirs pour la réalité. Enfin, l'armée succombait à deux autres « dissonances cognitives ", plus lourdes de conséquence puisque ce sont bien elles qui vont sceller le «fiasco de Kargil ». La première reposait sur l'idée que les Etats-Unis, craignant une guerre conventionnelle de grande envergure, voire la menace de l'utilisation de l'arme nucléaire, interviendraient en leur faveur ${ }^{83}$. Or, une telle attitude était doublement improbable : le département d'Etat avait répété à plusieurs reprises sa ferme hostilité à toute modification du statu quo au Cachemire et l'intervention au Kosovo se déroulant au même moment, il était inenvisageable qu'il se charge d'un autre dossier aussi sensible. Plus grave fut la sous-estimation étonnante du «facteur Sharif ». Ceci a de quoi surprendre compte tenu de la traditionnelle méfiance de l'armée à l'égard des civils et de sa bonne connaissance de la personnalité du premier ministre. Pourquoi avoir engagé une telle opération sans s'être assuré d'un soutien infaillible et d'un suivi politique du volet militaire ? La puissance du déni de réalité provient, rappelle Braud, de ce rempart défensif qu'il dresse contre l'inédit et contre un coûteux renoncement au système d'intelligibilité préalablement intériorisés4. Les ressorts psychologiques de l'illusion doivent donc être réinterprétés ici dans le cadre plus large du décalage entre cette interiorisation et le changement brutal intervenu dans les règles du jeu du rapport civils-militaires au Pakistan explicité précédemment. L'armée avait tant et si bien intériorisé son monopole sur la gestion des relations avec l'Inde, le traitement 
presque exclusivement militaire de ce dossier trop sensible pour être laissé à la charge de civils "corrompus » et incapables à défendre "l'intérêt national »" qu'elle n'a pu surmonter les perturbations cognitives introduites par ce mouvement d'autonomisation inédit du Premier ministre et son intrusion dans son «domaine réservé ». Le déni de réalité est tout aussi présent dans l'attitude du Premier ministre. Il se manifesta clairement dans la croyance qu'il était possible d'instrumentaliser à des fins personnelles une institution aussi puissante que l'armée - avec le seul soutien du clan familial, du directeur de l'ISI et d'un ou deux généraux reconnaissants. Il s'est encore révélé dans sa conviction qu'il serait aisé de lui faire avaler la reculade de Washington ${ }^{86}$ en l'accusant ensuite publiquement d'aventurisme, le tout sans avoir à déléguer la gestion de la crise au ministère des affaires étrangères et sans risquer son poste. "Soif mégalomaniaque et paranoïaque du pouvoir ", "personnalité indécise et inconstante ", "naïve et puérile », les jugements sévères de ceux qui l'ont rencontré sur les «pathologies» psychologiques de "l'acteur Sharif» abondent ${ }^{87}$. Elles sont toutefois d'un intérêt limité, difficiles à vérifier en pratique et très probablement exagérées à l'approche du coup d'Etat dont la justification passe justement par la mise en avant de la «dangerosité» du Premier ministre. Toutefois, si l'on admet l'importance du phénomène d'isolement des décideurs à l'origine des dénis de réalité, les calculs politiques risqués de Nawaz Sharif acquièrent une meilleure intelligibilité. A cet égard, les travaux de David Kipnis et de Irving Janis, se révèlent d'une grande utilité ${ }^{8}$. Kipnis montre ainsi que l'individu arrivé "aux sommets" a tendance à surévaluer le rôle de son intelligence et de son habilité au détriment des facteurs conjoncturels de cette ascension. Il souligne que plus la trajectoire est rapide et plus le sentiment d'exaltation de soi et d'«arrogance» est intense. Cette analyse est particulièrement pertinente dans le cas de Nawaz Sharif. Il a été catapulté en politique par l'armée, par le général Zia, à l'âge de 30 ans. Neuf ans plus tard -et après avoir occupé le poste déterminant de "chief minister» du Punjab - il était élu Premier ministre (en 1990), puis réélu sept ans plus tard. L'ascension politique fulgurante de cet industriel punjabi, patronnée initialement par l'armée, peut expliquer son excès de confiance, et surtout, sa mauvaise évaluation du rapport de force avec les militaires au cours de la crise de Kargil. Ceci était d'ailleurs visible dès octobre 98 quand il interpréta l'acceptation de démissionner du général Karamat comme une victoire personnelle. L'illusion se renforçait l'année suivante comme en témoignent ses assauts croissants à l'égard de l'armée, alors même que celle-ci envisageait à trois reprises des plans d'urgence pour prendre le pouvoir. Victime de cette illusion pendant la crise de Kargil, il se trouvait finalement contraint à «l'extraversion" de sa politique étrangère, à appeler les Etats-Unis à l'aide pour imposer à l'armée une décision dont elle ne voulait pas, en un mot à ce que Ghassan Salamé appellerait «l'appel d'empire » ${ }^{89}$. Son départ précipité pour Washington et la déclaration américano-pakistanaise de juillet est bien avant tout le résultat de l'incapacité du premier ministre à mener à son terme sa stratégie de déstabilisation de l'institution militaire. Janis quant à lui, souligne l'importance du contexte dans lesquels sont prises les décisions de politique étrangère : dans l'urgence ( «hot cognitive processes») et en cercle très restreint. Cela peut conduire à des fiascos spectaculaires ou à des décisions aux conséquences désastreuses, comme l'intervention de la Baie des Cochons en 1961. Tel fut le cas de la crise de Kargil. Dans un contexte d'urgence, entouré d'un cercle restreint de confidents et conseillers, disposant d'informations incomplètes ou fausses (car si Sharif a bien été informé des modalités de l'opération de Kargil, à quel degré de détails personne ne peut le dire), le 
décideur aura tendance selon Janis à mettre en œuvre des stratégies protectrices. Il cherchera tout d'abord à se défausser de sa responsabilité sur un tiers (ici l'armée "aventuriste»). Il sélectionnera ensuite de façon irrationnelle les informations (menace de guerre générale avec l'Inde évoquée par Sharif) qui pousseront à une décision en catastrophe (le voyage précipité à Washington où de l'avis d'observateurs bien placés, il a succombé à la panique). Ces variables psychologiques n'ont pu toutefois avoir un tel impact que parce qu'elles étaient sous-tendues par des facteurs d'ordre structurel. Ce qui détermina le fiasco de la crise de Kargil a été ce refus radical d'un premier ministre, engagé dans un rapport de force avec l'armée intenable dans le cadre $\mathrm{du}$ système politique pakistanais, d'en déléguer la gestion aux principales administrations publiques, mieux aguerries aux crises internationales que la coterie dont il s'était entouré, ainsi que son évaluation totalement erronée de ce rapport de force. La crise de Kargil a ainsi montré que, dans un Etat marqué par vingt-cinq ans de dictature militaire qui, selon Rashid, « ont contribué à miner les gouvernements civils avec son contrôle exclusif sur la politique étrangère vis-à-vis de l'Inde comme de $l^{\prime}$ Afghanistan /.../ et le rôle des services secrets militaires $»^{90}$, puis par dix années de démocratie dont la corruption a été la principale bénéficiaire ${ }^{91}$, la concertation entre décideurs civils et militaires et entre ceux-ci et la société, reste à inventer. Or, les conséquences de cette crise dépassent le seul cadre du Pakistan. En internationalisant les défaillances de son système politique, l'Etat pakistanais a miné les chances déjà faibles des Cachemiris de sensibiliser l'opinion mondiale au drame humain qu'ils vivent. Un conflit qui a fait 60000 morts en dix ans (militaires et civils), des centaines de milliers de réfugiés en Inde (musulmans comme hindous) et au Pakistan et transformé la Vallée de Srinagar en un Etat policier (200 000 membres des forces de sécurité indiennes y sont déployés, soit environ un pour chaque 20 habitants), s'est trouvé ramené à une affaire de délimitation territoriale et de "fondamentalisme islamique ».

\section{NOTES}

1. Je remercie le CERI pour avoir rendues possibles les deux enquêtes de terrain au Pakistan (en octobre 1998 et 1999) sur lesquelles sont basées les analyses qui suivent. Elles n'engagent toutefois que leur auteur.

2. Jehangir Karamat, « Learning from Kargil », The News (quotidien pakistanais), 5 août 1999. Il a été à ce poste, le plus haut élevé dans l'armée, de 1996 à 1998.

3. Cf. The Herald (mensuel pakistanais), mars 2000, p. 53.

4. Zaffar Abbas, «Whodunnit?», The Herald, août 1999, p. 67. Z. Abbas ajoute que "l'un des aspects les plus regrettables de cette opération de Kargil est que tout le monde hors du pays sait probablement quel était son sens ; le seul laissé dans l'obscurité est le peuple pakistanais » et les veuves des soldats « martyrs » de Kargil. 5. M. Lodhi, « The Kargil Crisis : Anatomy of a Debacle », Newsline (mensuel pakistanais), juillet 1999. Reconnue pour l'acuité de ses analyses et son franc-parler, 
M. Lodhi est également directrice de la publication d'un des principaux quotidiens anglophones du pays (The News).

6. Graham T. Allison, Essence of Decision. Explaining the Cuban Missile Crisis, Boston, Little, Brown, 1971.

7. L'ouvrage de Baghat Korany, How Foreign Policy Decision are Made in the Third World. A Comparative Analysis (Londres, Westview Press, 1986) est révélateur de ce rendez-vous manqué.

8. Ibid., p. 52.

9. Ahmed Rashid, « The Decision-making process in Pakistan : Implications for IndiaPakistan Relations », in Saeed Shafqat ed., Contemporary Issues in Pakistan Studies, Lahore, Azad, 1998, pp. 151-168, p. 152.

10. Pour Mariam Abou Zahab également, « le changement au sommet de l'Etat était devenu inévitable depuis la « débâcle » de Kargil ». M. Abou Zahab, « Islamabad : l'Armée du Salut ? », Politique internationale, n 86, hiver 99-2000, pp. 365-381, p. 365. 11. La « Line of Control » (LoC) est une ligne temporaire de délimitation qui va du glacier du Siachen, au nord, jusqu'à l'extrême sud du Cachemire (Manawar). A partir de là et jusqu'à l'extrémité sud-est (les Marais de Kutch), les deux pays sont séparés par une frontière internationale ( « working boundary »). Du côté indien, se trouve l'Etat du Jammu\&Cachemire (soit la vallée du Cachemire, le Jammu et le Ladakh) qui, originellement autonome, a été progressivement intégré pleinement à l'Union indienne. Du côté pakistanais, l'ancien territoire de l'Etat princier recouvre l'Azad («Libre ») Cashemire (lui aussi officiellement autonome mais en réalité totalement inféodé au gouvernement central) et les Territoires du Nord (sous administration directe d'Islamabad). Le tracé de la LoC a fait l'objet d'un accord entre l'Inde et le Pakistan en juillet 1972 et a remplacé de facto la Ligne de cessez-le-feu de janvier 1949. 600000 soldats, policiers et forces paramilitaires sont déployés du côté indien et 80000 soldats pakistanais le long de la LoC (cf. Rashid, op. cit., p. 151).

12. Entretien avec un ancien directeur des services de renseignement de l'Armée, Rawalpindi, novembre 1999.

13. Pour une défense de cette lecture institutionnaliste du soulèvement cachemiri, voir : Sumit Ganguly, The Crisis in Kashmir. Portents of War. Hopes of Peace, Cambridge, Cambridge University Press, 1997 ; Balraj Puri, Kashmir. Towards Insurgency, London, Sangam Book, 1993.

14. Pour les études les mieux documentées sur la revendication autonomiste comme fondement de l'identité cachemirie, voir : John Cockell, Ethnic Nationalism and Subaltern Political Process : Exploring Autonomous Democratic Action in Kashmir, Third Pan European Conference (ISA), Vienne, septembre 1998, non publié ; Victoria Schofield, Kashmir in the Crossfire, Londres, I.B. Tauris, 1996.

15. Stephen Cohen, The Pakistan Army, Oxford, Oxford University Press, 1998 (1ère édition, 1994), p. 145.

16. Il y aurait 60000 personnes déplacées en Azad Cachemire. Les tirs de l'été 98 ont donné lieu à l'un des plus importants déplacements de villageois dans la vallée de Leepa depuis la guerre de 1971 comme en témoigne la surpopulation du camp de Hatian où nous nous sommes rendus. Selon certains observateurs, pour inciter les gens à retourner dans leurs villages et ne pas avaliser la " politique de la terre brûlée » menée par l'Inde, les conditions de vie dans ces camps sont rendues particulièrement difficiles (comparées à celle prévalant dans les camps de réfugiés venant d'Inde qui peuvent disposer, par exemple, d'habitations en dur). 
17. J. Karamat, op. cit.

18. Cf. Rifaat Hussein, "The deal that never was », The News, 1er août 1999 ; Zaffar Abbas, op. cit.

19. Selon les termes du président Clinton dans une conversation téléphonique privée avec Sharif le 14 juin, rendue curieusement publique par les officiels américains. Lodhi, op. cit.

20. Samy Cohen, « Décision, pouvoir et rationalité dans l'analyse de la politique étrangère ", in Smouts (M.-C.) éd., Les nouvelles relations internationales. Pratiques et théories, Paris, Presses de Sciences-Po., 1998, p. 87.

21. Cette expression, utilisée par Ivo Duchacek dans le contexte fort distinct des Etats fédéraux, est ici tout à fait pertinente : « les Etats-nations [sont] comme des acteurs multivocaux /.../ audibles au-delà de leurs frontières souveraines tel un choeur souvent polyphonique, parfois cacophonique ». « Multicommunal and Bicommunal Polities and Their International Relations », in Ivo D. Duchaceck et al., Perforated Sovereignties and International Relations. Trans-Sovereign Contacts of Subnational Governments. New York, Greenwood Press, 1988, p. 4.

22. Il réunit le président de la République, le premier ministre, le ministre des affaires étrangères, le secrétaire de la défense et les chefs d'état-major des trois Armées (Terre, Aviation, Marine). Cf. M. Lodhi, op. cit.

23. Voir Amélie Blom, «The Kashmir politics of Pakistan », Pakistan in International Politics : Foreign Policy Making and Security Issues, Colloque du CERI, Paris, 7 décembre 1998, non publié.

24. Kotera M Bhimaya, Civil-Military Relations. A Comparative Study of India and Pakistan, Dissertation, Rand Graduate School, nd., p. 141.

25. Cohen, op. cit., p. 79.

26. Entretien, Islamabad, octobre 98.

27. Rashid, op. cit., p. 153.

28. Saeed Shafqat, Civil-Military Relations in Pakistan, Boulder, Westview Press, 1997, p. 169.

29. Cité in The Herald, mai 1999, p. 29.

30. Lodhi, op. cit.

31. Cet universitaire et journaliste pakistanais respecté, et décédé en 1999, est cité in The Herald, mai 1999, p. 31.

32. Rashid, op. cit., p. 154. Voir également, son ouvrage Taliban : Islam, Oil and the New Great Game in Central Asia, Londres, I.B. Tauris, 1999.

33. Vernon Hewitt, Reclaiming the past ? The search for political and cultural unity in contemporary Jammu and Kashmir, Londres, Portland Books, 1995, pp. 185-88. Notons toutefois que Hewitt se base sur The Kashmir Connection, document de discussion préparé par la House of Representatives Republican Task Force on Terrorism and Unconventional Warfare, Washington, mai 1994.

34. Pour un compte-rendu extrêmement bien documenté de cette crise, voir : Seymour M. Hersh, « On the Nuclear Edge », The New Yorker, 29 mars 1993, p. 56-73.

35. Ses visites, nombreuses et rapprochées, au quartier général de l'Armée et de l'ISI à partir de janvier 1999 indiquent qu'il n'a pu être mis devant le fait accompli. Voir les révélations de l'ex COAS Aslam Beg in Abbas, op. cit.

36. Abdul Hayee, « Ms Bhutto's interview », Nation (quotidien pakistanais), 14 août 1999. 
37. Précisons que les seules sources sont ici les participants pakistanais à ces discussions : un lieutenant-général à la retraite et l'ancien secrétaire d'Etat aux Affaires étrangères, Niaz Naïk (dans des confidences faites à des journalistes). L'un de ces participants date ces négociations secrètes antérieurement à la crise de Kargil, l'autre à son tout début.

38. Cf. Rifaat Hussein, op. cit. L'armée n'était pas a priori hostile à une retraite mais elle attendait de Sharif qu'il temporise jusqu'à début septembre (les premières neiges rendraient alors la zone impraticable) et obtienne des concessions diplomatiques (la démilitarisation du Siachen ou même juste un désescalade le long de la LoC d'Azad Cachemire).

39. Les révélations de Sonia Gandhi sur le "Sugargate » (un accord entre le Army Welfare Trust of Pakistan, alors dirigée par un fidèle de Sharif, et la Kundan Rice Mills indienne, en février 99 aurait permis l'exportation de sucre pakistanais à hauteur de 225 millions de dollars et dégagé un bénéfice de 40 millions de dollars pour les huit entreprises sucrières dirigées par la famille de Nawaz Sharif et ses amis) n'ont été que mollement démenties par Vajpayee. Newsline, octobre 1999.

40. Cité dans Newsline, juillet 1999.

41. M. Abou Zahab, op. cit., p. 379.

42. Robert Putnam, « Diplomacy and Domestic Politics : The Logic of Two-Level Games », in Peter Evans, Harold K. Jacobson, Robert D. Putnam ed., Double-Edged Diplomacy. International Bargaining and Domestic Politics, Berkeley, University of California Press, 1993, p. 431-468, p. 434.

43. Dans une enquête effectuée en 1996, les forces armées arrivaient en tête des institutions auxquelles les sondés accordaient leur confiance « pour dire la vérité et faire le bien du pays » (plus de $80 \%$... contre $13 \%$ aux partis politiques). Cf. Riaz Hassan, Faithlines : religion, society and the state in Indonesia and Pakistan, paper présenté au «International Seminar on Western Perceptions of Muslim; Muslim Perceptions of the West », International Islamic University, Islamabad, 4-6 octobre 1997.

44. Les dépenses militaires représentent en effet un tiers du budget de l'Etat (le remboursement de la dette extérieure en absorbant $56 \%$, les difficultés économiques endémiques du pays ne sont pas difficiles à comprendre). Juste avant Kargil, l'armée avait obtenu une hausse de $11 \%$ de ses crédits.

45. Nous les qualifions ainsi car leur raison d'être est de mener la lutte armée, qualifiée de «jihad", sur tous les fronts où les musulmans sont selon eux opprimés (et en priorité au Cachemire). Ils se distinguent tout à la fois des partis islamistes constitutionnalistes pakistanais (auxquels ils sont par ailleurs liés) et des guérilleros cachemiris d'inspiration séculière (comme la branche armée du JKLF).

46. Il est bien sûr très difficile d'avoir une évaluation fiable du nombre total de leurs combattants. Le chiffre cité est proposé sur la base d'une évaluation à la baisse de celui de 500000 avancé par le principal parti islamiste pakistanais, le Jama'at-i Islami, (entretien, Mansoora, novembre 1999) qui semble excessif si l'on considère que le Lashkar-e Taïba, l'une des grandes organisations jihadistes, réunit 200000 participants à sa dernière convention (tous n'étant pas des mujahidin).

47. Amélie Blom, «Les partis islamistes à la recherche d'un second souffle », in Christophe Jaffrelot ed., Le Pakistan, carrefour de tensions régionales, Bruxelles, Complexe, 1999, pp. 99-115.

48. Le Harkat Ul-Ansar, renommé Harkat Ul-Mujahidin après avoir été inclus à la liste des groupes terroristes du département d'Etat américain, comptait des sympathisants 
parmi les officiers organisateurs de la tentative de coup d'Etat de 1995 au Pakistan. Or les liens entre les putschistes et le Harkat, ni même le fait que leurs armes provenaient $\mathrm{du}$ «jihad» au Cachemire, ne sont apparus dans le jugement de la Cour martiale. On peut aussi mentionner que c'est pour obtenir la libération d'un de ses militants emprisonné au Cachemire indien qu'a été organisé le détournement de l'avion d'Indian Airlines, de Katmandou à Kandahar en décembre 1999, mettant le Pakistan dans une position si délicate qu'il a refusé leur atterrissage à Lahore.

49. Selon le dirigeant du United Jihad Council (organe de coordination de 14 groupes jihadistes) créé au début des années 90 et actuellement dominé par le JI), cité dans The Herald, juillet 1999.

50. Sumantra Bose, The Challenge in Kashmir. Democracy, Self-Determination and a Just Peace, Londres, Sage, 1997, p. 59.

51. Chef d'Etat major indien cité dans The Nation, 27 octobre 1999. Le principal organe politique représentant les militants autonomistes cachemiris, le APHC, avance lui le chiffre de 20000 soldats indiens tués dans les dix dernières années.

52. De l'aveu même du chef d'Al-Badr Mujahidin.

53. La déclaration de Washington stipule en effet que « Le président Clinton et le premier ministre Nawaz Sharif /.../ sont d'accord sur le fait qu'il est vital à la paix du sous-continent que la Ligne de Contrôle au Cachemire soit respectée par les deux parties selon les accords de Simla de 1972. Il a été décidé /.../ que des actions concrètes seront prises pour la restauration de la LoC /.../ Le premier ministre Sharif accepte que le dialogue bilatéral qui a commencé à Lahore en février constitue le meilleur forum pour résoudre toutes les sujets de contentieux entre l'Inde et le Pakistan, le Cachemire y compris ». Texte intégral reproduit in Lodhi, op. cit.

54. Lodhi, op. cit.

55. Voir le compte-rendu de leurs déclarations in Abbas, op. cit.

56. Il est à cet égard significatif que les mouvements jihadistes cachemiris aient été évoqués lors de la première visite d'un officiel de haut niveau américain au Pakistan après le coup d'Etat du 12 octobre, Michel Sheean, coordinateur de la lutte antiterroriste au département d'Etat.

57. Le APHC a été créé en 1992 ou 1993 à Srinagar pour coordoner la lutte d'une trentaine d'organisations politiques, religieuses et sociales. Les principales sont : le Jamaat-i Islami de Syed Ali Shah Gilani, la People's Conference de Abdul Ghani Lone, la People's League de Shabir Ahmad Shah et Cheikh Abdul Aziz, le JKFL de Yasin Malik, le Awami Action Committee de Mirwaiz Omar Farooq, la Muslim Conference de Abdul Gani, et le Ittehad-ul-Muslimeen de maulana Abbas Ansari (composition en 1994). Le bureau central du APHC est à Srinagar mais il a aussi plusieurs bureaux au Pakistan (à Muzaffarabad et Islamabad entre autres).

58. Entretien, Islamabad, novembre 1999.

59. Cité dans Dawn (quotidien pakistanais), 24 octobre 1999.

60. Son appel au boycott des élections législatives indiennes de 99 a été majoritairement suivi si l'on en croit le chiffre de $17 \%$ de participation à celles-ci dans la Vallée de Srinagar.

61. Selon Cockell, op. cit. et Schofield, op. cit. Pour le Pakistan, voir le sondage paru dans The Herald Annual, janvier 1997. Dans les villes des régions du Pakistan ellesmêmes traversées par des tendances autonomistes (Karachi, Sukkur, Quetta), l'option de l'indépendance est majoritaire. Plus surprenante est la réponse des Lahoris, 
traditionnellement favorables au rattachement du Cachemire au Pakistan : ils sont désormais un tiers à admettre l'indépendance comme la meilleure solution.

62. Bose, op. cit., p. 63.

63. Déjà en 1995, 70 \% des personnes interrogées dans un sondage réalisé par le magazine indien Outlook (18 October 1995) affirmait désapprouver la progression du fondamentalisme islamique au Cachemire. Le résultat de ce sondage réalisé dans un contexte de guerre civile doit être apprécié avec une extrême prudence. Il est cependant la seule source de ce type disponible.

64. Arif Jamal, « Camping for jihad », The News, 14 août 1999.

65. Jihad Times (hebdomadaire en ourdou du mouvement), 13 novembre 1999.

66. Chiffre donné par le Kashmir Action Committee, entretien, Lahore, novembre 1999.

67. Putnam, op. cit., p. 436.

68. Sa prise en compte se fait encore à la marge en Relations Internationales - car on ne saurait confondre les affects avec les seules perceptions et « misperceptions ", thème quant à lui bien investi par la discipline.

69. Extraits des enregistrements des réunions à la Maison-Blanche réalisé par Kennedy du 18 au 26 octobre 1962, Le Monde, 15 août 1997, pp. 10-11.

70. Philippe Braud, L'émotion en politique, Paris, Presses de Sciences-Po., 1996, pp. 8-9.

71. Ibid.

72. Cf. Jahan Dad Khan, Pakistan Leadership Challenge, Oxford, Oxford University Press, 1999, pp. 222.

73. Au seul Punjab il est vrai. Le Cachemire est en effet un sujet de préoccupation surtout dans cette région limitrophe du Cachemire (et en Azad Cachemire) qui est toutefois la plus peuplée du pays et dont est issue la majorité des décideurs militaires comme civils.

74. Ainsi que le suppose Olivier Roy qui y voit l'objectif central de la politique « agressive » du Pakistan au Cachemire (à l'instar de Afghanistan). O. Roy, « Pakistan : un nouvel « Etat-voyou? », Politique internationale, n 86, hiver 99-2000, pp. 383-399, p. 399.

75. Tilly (C.), Contrainte et capital dans la fondation de l'Europe 990-1990, Aubier, 1990. 76. Voir Mushirul Hasan, Nationalism and Communal Politics in India, Delhi, Manohar, 1991 (1ère éd., 1979).

77. Denis-Constant Martin, «Identités et politique : récit, mythe et idéologie », in D.-C. Martin éd., Cartes d'identité. Comment dit-on « nous » en politique ?, Paris, Presses de Sciences Po., 1994, pp. 13-38.

78. «Il est toujours possible d'unir les uns aux autres par les liens de l'amour une plus grande masse d'hommes, à la seule condition qu'il en reste d'autres en dehors d'elle pour recevoir les coups. /.../ Je me suis occupé jadis de ce phénomène que justement les communautés voisines et même apparentées se combattent et se raillent réciproquement ; par exemple Espagnols et Portugais, Allemands du Nord et du Sud, Anglais et Ecossais, etc. Je l'ai appelé Narcisissisme des petites différences ». Sigmund Freud, Malaise dans la civilisation, Paris, PUF, 1971 (1ère éd. 1929), p. 68.

79. Entretien, Rawalpindi, octobre 98.

80. Pontalis (J.-B.), Ce temps qui ne passe pas, Paris, Gallimard, 1997.

81. Shafqat, op. cit., p. 173 ; Ian Talbot, « The Punjabisation of Pakistan. Myth or Reality ?, « Pakistan, fifty years after independence », Colloque du CERI, op. cit.

82. Lodhi, op. cit.

83. Entretien avec un lieutenant-général à la retraite, Islamabad, octobre 99. 
84. Braud, op. cit., p. 190.

85. Voir les entretiens menés par S. Cohen, op. cit., pp. 59-63.

86. D'autant plus difficile à avaler si l'on en croit les témoignages selon lesquels Sharif se serait engagé auprès des militaires avant son départ à obtenir des concessions, ou tout du moins, que la LoC ne soit pas mentionnée dans la déclaration finale.

87. Entretiens, Islamabad, Rawalpindi, octobre 1999.

88. David Kipnis, The Powerholders, Chicago, Chicago University Press, 1976 ; Irving Janis, Decision-Making : a Psychological Analysis of Conflict, Choice and Commitment, New York, Free Press, 1977.

89. G. Salamé, Appels d'empire. Ingérences et résistances à l'âge de la mondialisation, Paris, Fayard, 1996.

90. Ahmed Rashid, « Pakistan : Civil versus Military », Current History, Janvier 2000.

91. Les seuls chefs de l'exécutif élus depuis 1988 sont tous deux aujourd'hui sous le coup de poursuites judiciaires pour détournement des fonds publics (Benazir Bhutto vit en exil et Nawaz Sharif est détenu à la prison de Karachi, accusé d'avoir comploté l'assassinat de son principal rival dans la crise de Kargil, le général Musharraf).

INDEX

Index géographique : Pakistan

Mots-clés : crises politiques, politiques publiques, Relations Internationales

Index chronologique : 1999 